\title{
Optical Phenomena in Mesoscale Dielectric Particles
}

\author{
Oleg V. Minin ${ }^{1}$, Igor V. Minin ${ }^{1, *}$
}

1 Tomsk Polytechnic University; ovminin@tpu.ru

* Correspondence: ivminin@tpu.ru; prof.minin@gmail.com

\begin{abstract}
During the last decade, new unusual physical phenomena have been discovered in studying the optics of dielectric mesoscale particles of an arbitrary three-dimensional shape with the Mie size parameter near $10(q \sim 10)$. The paper provides a brief overview of these phenomena from optics to terahertz, plasmonic and acoustic ranges. The different particle configurations (isolated, regular or Janus) are discussed, and the possible applications of such mesoscale structures are briefly reviewed herein in relation to the field enhancement, nanoparticle manipulation and super-resolution imaging. The number of interesting applications indicates to a new promising scientific direction emerged in optics, terahertz and acoustic ranges, and plasmonics. In this paper we present the authors' view of these problems.
\end{abstract}

Keywords: Mesoscale particle; Mie size parameter; optical effect

\section{Introduction}

Mesoscale dielectric particles are mesostructures, both single wavelength-scaled particles and chain or array formed of these particles, made out of relative low-refractiveindex (known as refractive index less than 2) dielectric low loss materials. The phenomenons associated with wavelength-scaled dielectric particles arise due to illumination field penetration in and concentration inside or outside near the shadow surface the particles of arbitrary 3D shape. This is different from plasmonics where radiation concentrates at the metal nanoparticle-environment interface. Mesoscale dielectric photonics aiming at the manipulation of strong optically induced electric and magnetic structured fields in and near dielectric wavelength-scaled structures with refractive index near two. Importantly, various three-dimension geometries and degrees of freedom can be employed to flexible control the structure and characteristics of such localized fields.

The exact solution of the Maxwell equation for plane wave scattering over a homogeneous dielectric sphere is described by the Mie equations [1], which include the size parameter $q=2 \pi R / \lambda$, where $R$ is the sphere radius, $\lambda$ is the emission wavelength depending on the refractive index $n$ of the particle. The field structures with the size parameter $q$ $\sim 10$ lying between the nano $(q \sim 1)$, wave and geometric optics $(q>100)$ have not been studied so far. But in the last decade, new, unusual phenomena have been discovered in optics of an arbitrary three-dimensional dielectric particles at the mesoscale level and gratings with $q \sim 10$ parameter. It is important to identify these phenomena. Below we present the authors' view of these problems.

\section{Optical optical whirlpools, nano-vortices, optical hearts}

The anomalous light scattering possesses the number of interesting and unusual properties such as a complex structure of the near field, which may include optical vortices, optical whirlpool and other specific traits of the nanoscale level [2]. For example, the term optical whirlpool was introduced in [3] by Bashevoy et al., who show that near the plasmonic 
resonance of the metal particle, nanosized optical vortices are observed, which resemble an optical whirlpool. Luk'yanchuk et al. [4] describe the optical vortices with the specific heart size, significantly lower than the diffraction limit for particles with a low size parameter $q<<1$. It is also found that at points matching the symmetric quadrupole resonance and forward and back scattering, near-field optical vortices appear.

Another interesting effect connected with the optical vortices, is a possibility of threedimensional subwavelength confinement of light outside the dielectric particle with $q \sim 10$ parameter, known as a photonic jet. This is associated with the vortex formation in the near field, namely the field squeeze due to the optical vortices occurred at the particle boundary, near the formation of the photonic jet [5].

Based on the Lorenz-Mie theory, it was found in [6], that spherical mesoscale particles with definite size parameters could cause substantially great field strength in singularities and form two extremely hotspots near the particle poles. Thus, the increase in the field intensity in the hotspots corresponded to 438 and 514 values for the size parameters of $q=22.24159$ and $q=28.64159$ of Teflon sphere, respectively. Also, a surprisingly large circulation was discovered for the three-dimensional Poynting vector in the form of heart (see Fig. 1), which was impossible to predict by a conventional two-dimensional analysis.

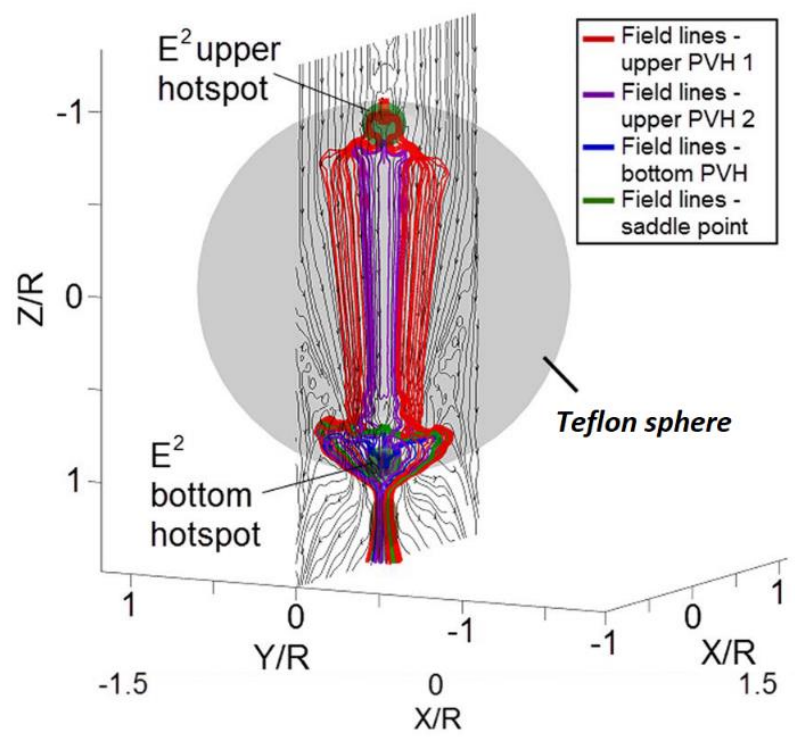

Fig.1. Three-dimensional view of Poynting vectors at critical points. Particle size parameter: $q=22.24159$. PVH - Poynting vector hotspot. Adapted from [6].

These new physical phenomena allow explaining such interesting effects as the formation of the photonic jet and hotspots.

\section{Fano resonances in dielectric Mie resonance mesophotonics}

The crucial role in the formation of Fano resonances play magnetic dipole resonances of isolated dielectric particles. The magnetic dipole mode of the dielectric particle excited at the wavelength of the magnetic resonance, can be stronger than the electric dipole response, thereby contribution to the scattering effectiveness. Luk'yanchuk et al. [7] show that lowscattering mesoscale dielectric microspheres can produce high-order Fano resonances associated with the internal Mie modes. It follows from this that the regions associated 
with such a superresonance can be located inside a spherical particle and the field structure has no WGM structure. These resonances observed at a certain size parameter, yield the magnetic field enhancement of the order of magnitude of $10^{4}-10^{7}$. In this respect, superresonances demonstrate the appearance of the magnetic photonic jets and giant magnetic fields in dielectric microspheres with high refractive index (about $n=4$ ), that may be attractive to many photonic applications.

Yue et al. [8] identified the super-enhancement focusing of Teflon spheres with the certain size parameters and the high refractive index lower than 2, which provided the field strength of about 4000 times stronger than that of the downward radiation and demonstrated the possibility of overcoming the diffraction limit despite the high sensitivity to losses in the particle material. That Teflon spheres were characterized by a unique arrangement of hotspots at the poles of the sphere and were stipulated by a specific behavior of the Mie modes [8]. The manifestation of the superresonance effect is clearly seen in Fig. 2. This figure also shows the effect of transformation of hot spots at the poles of the sphere in the absence of losses in the photonic jet when small losses are introduced into the material of the sphere. With increasing losses in the sphere material, the maximum intensity of the electric and magnetic fields was compared for the size parameter $q=22.24159$. At $q=28.64159$, the magnetic field intensity was approximately 2 times higher than that of the electric field, in the absence of losses in the in the particle material. The effect of a decrease in the size of the field localization (hotspots) down to less than the diffraction limit $(<\lambda / 2 n)$ was found when small losses are introduced into the sphere material, which can be even less than in the ideal case of the particle material without losses (Fig. 2(b)). 

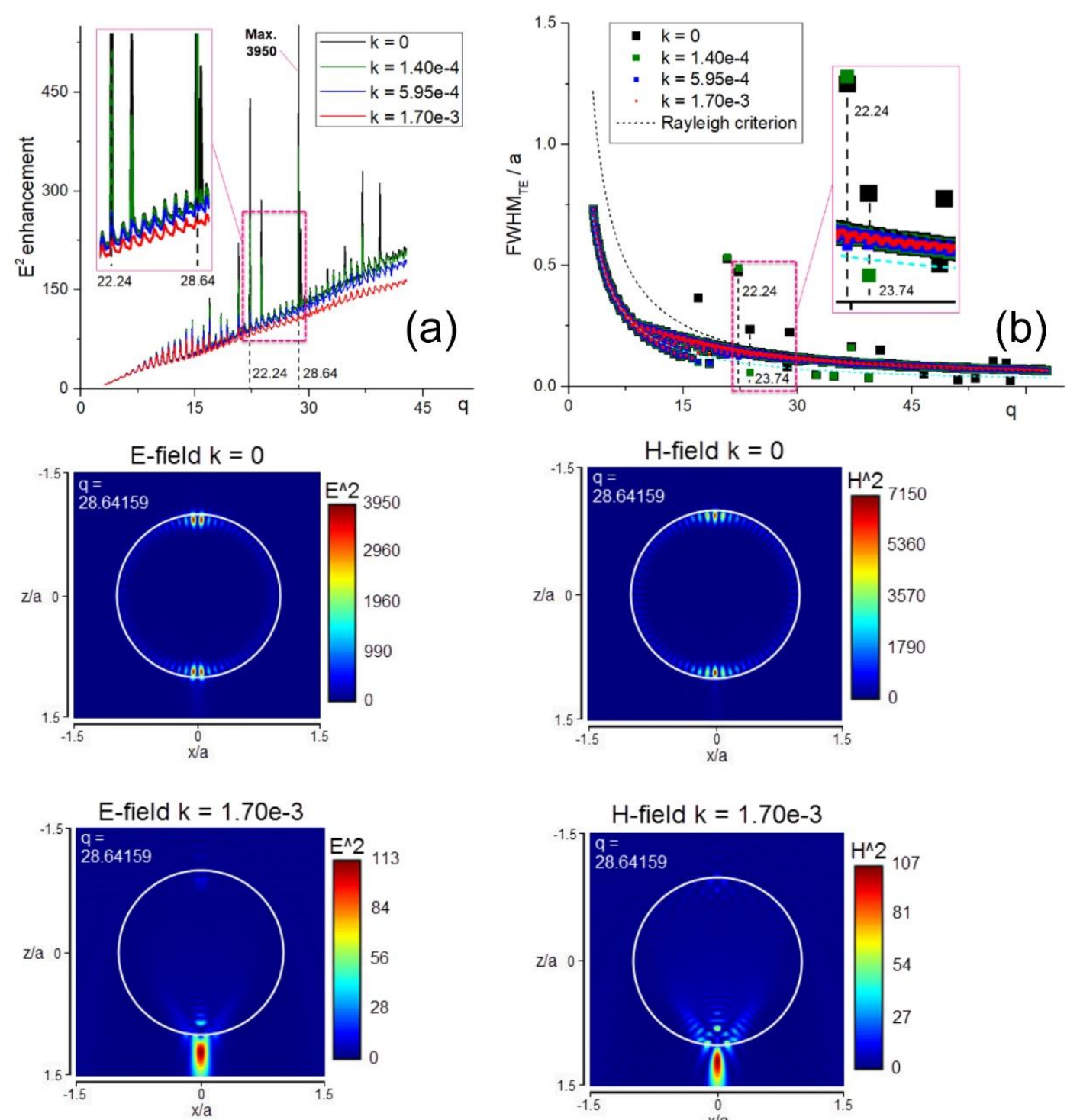

( c )

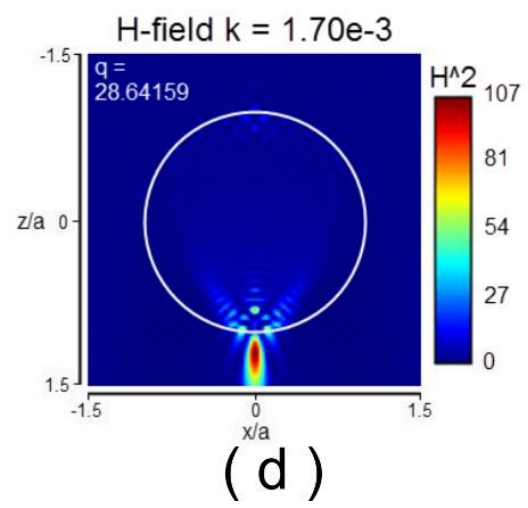

Fig. 2. Distribution of the maximum field intensity for a spherical Teflon particle (refractive index $n=1.43$ ) depending on the size parameter $q$ at various values of the absorption coefficient in the particle material k (a). Dependence of the width of the field localization region on the size parameter $\mathrm{q}$ for different values of the absorption coefficient in the material of the particle $\mathrm{k}(\mathrm{b})$. The intensity of the electric (c) and magnetic (d) fields for the resonance value of the parameter $\mathrm{q}=$ 28.64159 for the absorption indices $\mathrm{k}=0$ and $\mathrm{k}=1.7 \times 10^{-3}$. Adapted from [8].

\section{Giant magnetic field generation in mesoscale particles}

A new physical effect is the optical super-resonance in mesoscale dielectric spheres, which is conditioned by the high-order Fano resonance $[7,8]$ and can become a new method of achieving super-high magnetic fields. This conditioned by the following factors. The high-order Fano resonances in such particles are characterized by the high degree of the field localization, that exceeds the diffraction limit both inside the particle and on its surface. The latter is associated with the formation of regions having high values of the local wavenumber vectors by analogy with the superoscillation effects $[9,10]$. In accordance with this theory, the local wavenumber vector is a local phase gradient, viz. KI $=\Phi=\nabla E / E$. The high $K_{1}$ values can be created, for example, in free-space optics, including vortices and point vortices. This results from the uncertainty principle $\Delta E \Delta t \geq \hbar / 2$, which 
in terms of the number of photons and photon phase, can be reformulated as $\Delta N \Delta \Phi \geq 1 / 2$. Differentiation of this relation results in $K_{1} \sim \nabla N / N^{2}$, i.e., the high wavenumbers can be reached in the vicinity of the optical vortex representing a singularity (zero-intensity point) with the phase of the field circulating round this point [7]. For example, for superresonance condition (Fig.2) the local ratio of wave vectors in singular points near the outer surface of a particle can reach $K_{0} / K_{1} \sim 10^{-2}$, where $K_{0}$ is a wavevector of incident wave. So the giant magnetic fields can be created inside the dielectric particle due to creating subwavelength optical vortices with large phase gradients in the vicinity of singularities.

Optical vortices occur in dielectric mesoscale particles, when the size parameter $q$ exceeds a certain value depending on its high refractive index [5]. Accordingly, the ring currents create magnetic fields in accordance with the Biot-Savart law. Also from this law it is followed that to increase the amplitude of the $\mathrm{H}$ field one need to reduce the radius of the coil. Thus, the minimum radius of the correspondent equivalent coil is limited by the order of the wavelength. Inside the dielectric particle, the magnetic field can be enhanced by more than 4 orders, resulting in the magnetic field of about $10^{5}$ tesla, which approaches to the interatomic magnetic fields [7]. The disadvantages of high magnetic fields generation by higher order resonances are strong sensitivity to the losses in the mesoscale particle material. Note that such a magnetic field is comparable with magnetic cumulative generators [11,12]. With such magnetic fields, magnetic nonlinear optics effects can be expected, when a change in the refractive index is caused by the magnetic effects only. This magnetic nonlinear optics can however be implemented under two conditions: 1) rather low dissipation and 2) magnetic nonlinear response significantly exceeds the electric nonlinear response due to nonlinearity, viz. $\varepsilon=\varepsilon(E)$ [7].

An effect similar to the resonances noted above is also observed for particles of a different shape, other than a sphere or a cylinder. For example, for a cubic particle with a side of about $2 \lambda(\mathrm{q} 2 \pi)$ and $\mathrm{n}=1.59$, for linear polarization and under conditions of geometric resonance, the hot spot near the shadow side of the cube has an intensity $\left(E^{2}\right)$ about 400 higher than the intensity of the plane wave incident on the cube (for sphere with size parameter $\mathrm{q} \sim 5.38$, at a multiple overlap of resonant modes, $\mathrm{E}^{2}(\max ) \sim 40$ ). It could be noted that in dielectric mesoscale particles the maximum of field intensity is close to the particle boundary in contrast to plasmonic particle, where the maximum of the field intensity is at its surface. However, a detailed analysis of the electric and magnetic fields structures for non-spherical particles are beyond the scope of the paper and will be discussed elsewhere.

These effects of high-order Fano super-resonances is very attractive for such promising directions as, for example, the improvement of absorption effects, ablation induced by the magnetic pressure, and others.

\section{Photonic nanojets}

The term photonic nanojet was introduced by Chen et al. [13], although this effect was known before, e.g., [14-17]. In accordance with the theory of scattering on a cylindrical or spherical particles, e.g., [14, 18], the optical field both inside and outside the lowabsorbing particle illuminated by a light wave, is characterized by the spatial focus areas, 
called inner and outer focuses. These are associated with the surface curvature of the spherical particle leading to deformations of the phase wavefront incident on the particle. In this case, the spherical or cylindrical particle plays the role of a refractive lens, which focuses the light wave within the subwavelength volume [19]. At the same time, if the particle is in the converging wave area, the focusing area shifts toward the particle center and can be even locate inside it [20]. Moreover, in illuminating the particle by the structured fields, for example, a circularly polarized vortex beam, a reverse energy flow is observed in the forming photonic jet in the focal plane, that depends both on the topological charge of the optical vortex and the size parameters of spherical particles [21]. The complex interaction between the aberrated areas of the wavefield in the dielectric particle depends on the particle shape, its material properties, Mie size parameter, etc. At a determined proportion of the particle parameters (optical contrast, size parameter), one of the inner focuses of the particle moves to its outer boundary, thereby forming a strongly localized field, namely photonic jet, near the shadow-side surface and outside the particle. Chen et al. [13], reported that the photonic jet can propagate to a distance exceeding the wavelength $\lambda$ after its exit from the dielectric cylinder or microsphere with the diameter over $2 \lambda$, fabricated without material loss [22]. The photonic jet is characterized by the beam waist can be lower than the classical diffraction limit [19], namely about one third of the illuminated light wavelength. Note that in flat diffraction optics, such a size of the beam waist is achieved at a focal length less than the wavelength [23, 24]. But in general case, the transverse size of the photonic jet can be either smaller or larger than the diffraction limit. It should be noted that the photonic jet occurs in the space region, where near-field scattering plays a significant part (evanescent fields). Therefore, the agreement between the geometric optics approximation and the strict electromagnetic theory is less accurate for the particle size within the order of the light wavelength since the evanescent field is neglected in geometric optics [25]. The near-field region usually locates at distances not exceeding several particle diameters and is characterized by a notable contribution of the radial component of the optical field to this region. In turn, this condition restricts the size range of the dielectric particles, which is of the order of several wavelengths and even equals the light wavelength $[5,26]$, i.e., has mesoscale sizes. Hence, the specific value of the incident wavelength is not critical until mesoscale conditions are true.

The phenomenon of the photonic jet is not specific to dielectric mesoscale axisymmetrical 2D (cylindrical) or 3D (spherical) particles. In [27, 28], it was shown for the first time that the protonic jet can be formed by nonspherical and asymmetrical dielectric mesoscale particles of an arbitrary shape (Fig.3(i)), both in transmission and reflection modes. This provides better understanding of the photonic jet properties and possible ways of adjustment of such structured wave beams. On the other hand, the particle axial asymmetry and nonsphericity cause new optical effects. In particular, the focusing properties of mesoscale cubic particles can be explained by the phenomenon of diffraction at the edge, and the propagation of light breaking the laws of classical geometric optics, in contrast to the refractive phenomena for spherical particles [26]. 

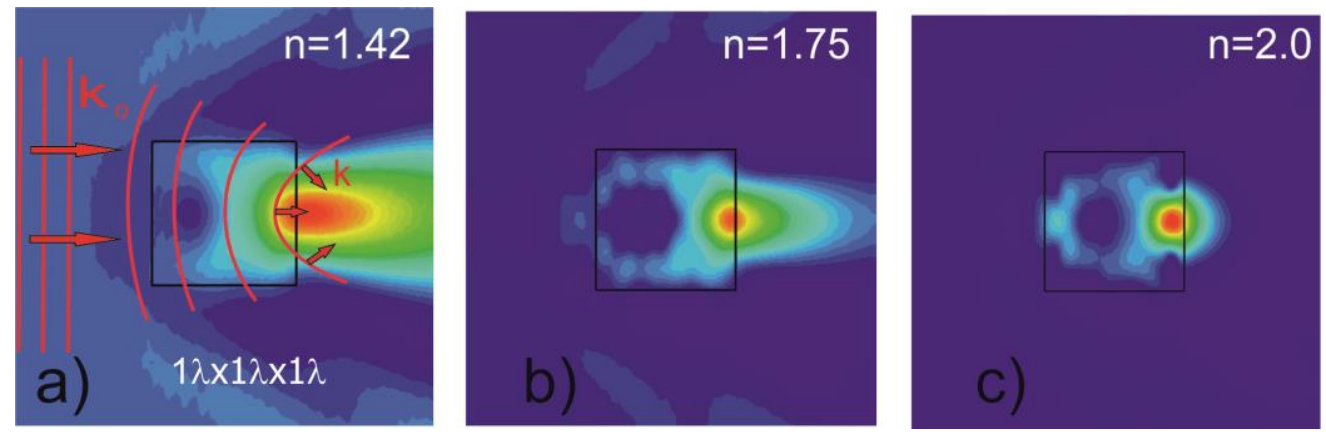

Fig.3(i). The dependences of electric field intensity $\left(E^{2}\right)$ localization area vs refractive index contrast of $n=1.42(\mathrm{a}), 1.75(\mathrm{~b}), 2.0$ (c) for dielectric cube with one wavelength dimensions. In (a) as example a wavefront curvature distortion is show due to the continuity of the tangential component of the E field in the air and in the cubic particle. $\mathrm{K}$ is a wavefront vector.

A range of the photonic jet applications is currently rather wide. Since the early 2000s, it has been used for optical manipulations [29] and can also be applied to detect and manipulate the biological objects [30], biomedicine [31,32], micro and macro manipulations [33], optical tweezers [34, 35], Raman spectroscopy [36, 37], microscopy [38,39] and many others.

\subsection{Photonic jet: electric or magnetic?}

It is interesting to note, that for dielectric particles with the relative large size parameters, the photonic jet is rather magnetic than electric [5]. In other words, during the formation of the photonic jet, the magnetic field enhancement can be stronger, than that which corresponds to the electric field. Figure 3(ii) [5] depicts the electric-field $E^{2}$ distribution with the field strength $H^{2}$ and the Poynting vector field module $S$ for a spherical particle the high refractive index $n \sim 1.5$ and size parameter $q \sim 10$. One can see that in the localized radiation area, the maximum magnetic-field intensity is about 2 times higher than the electric-field intensity.
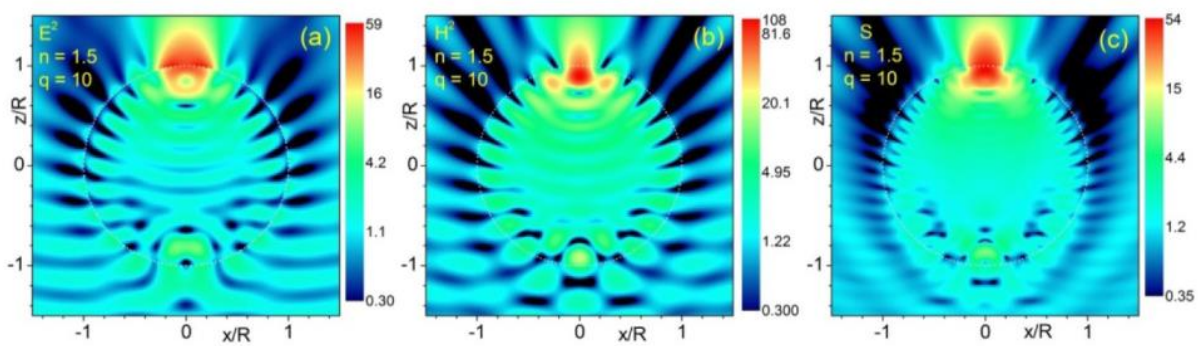

Fig. 3(ii). Intensity distribution in $\{x-z\}$ plane: electric field $E^{2}(a)$, magnetic field $H^{2}(b)$, Poynting vector field module $S$ (c) for spherical particle $(n \sim 1.5$ and $q \sim 10)$. Adapted from [15].

Usually, the electric dipole transitions are $10^{4} \sim 10^{5}$ stronger than the magnetic dipole transitions. But unlike plasmonic particles, the first resonance of the dielectric spherical particles, is a magnetic dipole resonance, which occurs when the light wavelength in the particle equals the diameter, viz. $\lambda / n \sim 2 R$. In this case, the electric field polarization is antiparallel at the opposite boundaries of the sphere, that provides a strong connection with circulating displacement currents, whereas at the center, the magnetic field vibrations are directed up and down [40]. 
Figure 3(iii) depicts the temporally averaged values of the electric $(a, b)$ and magnetic $(c, d)$ energy densities for a cubic Teflon particle with the refractive index $n \sim 1.45$ and size parameter $q \sim 2 \pi$ (cube dimensions are $2 \lambda \times 2 \lambda \times 2 \lambda$ ).

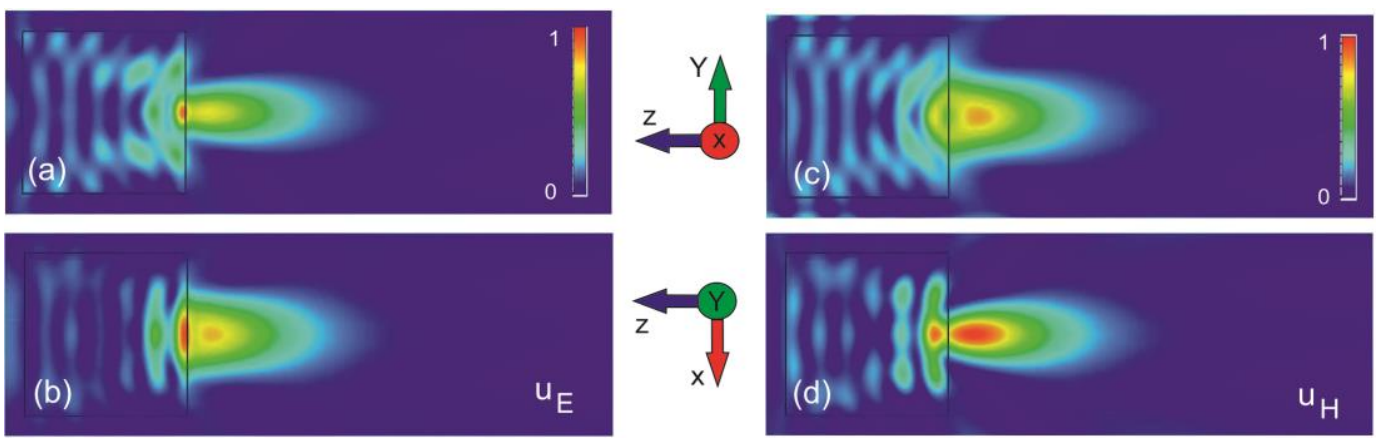

Fig. 3(iii). The temporally averaged normalized values of the electric $\mathrm{uE}_{\mathrm{E}}(\mathrm{a}, \mathrm{b})$ and magnetic $\mathrm{uH}_{\mathrm{H}}$ (c,d) energy densities for a cubic Teflon low loss particle illuminated by a plane wave with linear (Ex) polarization.

One can see that the maximum of the electric energy density is located inside the particle, near its shadow surface, while the maximum of the magnetic energy density is in the region of the photonic jet. In this case, the photonic jet is magnetic rather than electric.

The magnetic field enhancement earlier identified for nanoparticles with the high refractive index $[41,42]$, can therefore be observed for particles with the relatively low $(n<2)$ refractive index, if their size is larger than the wavelength $(R>\lambda)$, which is typical for the range of the photonic jet existence [5] for polarized [15] and unpolarized [43] illuminating light source. This property can be used, for example, for the enhancement of the radiation with the magnetic dipole transition [44].

\section{Anomalous apodization effect}

It is known that the amplitude mask apodization of the lens center reduces the transverse size of the focused beam, but the intensity in the focus lowers owing to blocking the part of the incident wave [45]. The same is also observed for spherical mesoscale particles [46, 47].

The anomalous apodization effect (size reduction of the photonic jet beam waist at its intensity increase) was first discovered for mesoscale particles of various nonspherical shape [48]. The effect was that the mask introduction in the irradiated particle surface led to a smaller number of optical vortices near the shadow-side surface of the particle. The mask apodization also increased the effective numerical aperture of the particle-lens, that was equivalent to the increase in the refractive index of the particle material [49-51]. It was also experimentally verified by a scale model using a copper-masked Teflon cubic particle (solid immersion lens) with $2.75 \lambda$ side length under radiation of a plane wave with $35 \mathrm{GHz}$ frequency ( $\lambda=8 \mathrm{~mm}$ wavelength) [51]. The lateral resolution and peak intensity enhancement of the produced photonic jet increased by about $36.4 \%$ and $36.0 \%$ in this approach, respectively. The effect of anomalous apodization illustrated in Fig. 4(i). 


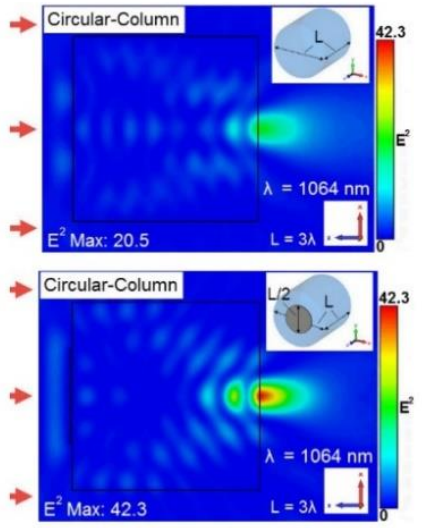

a)

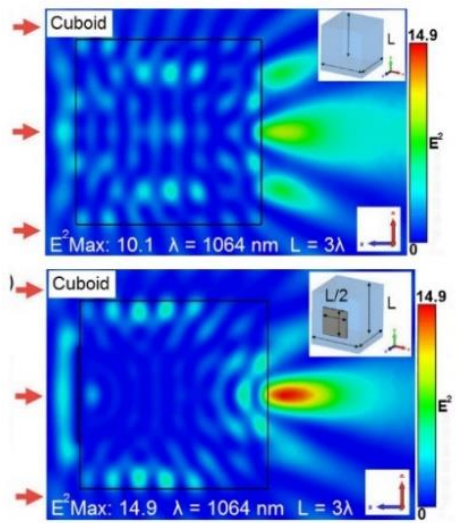

b)

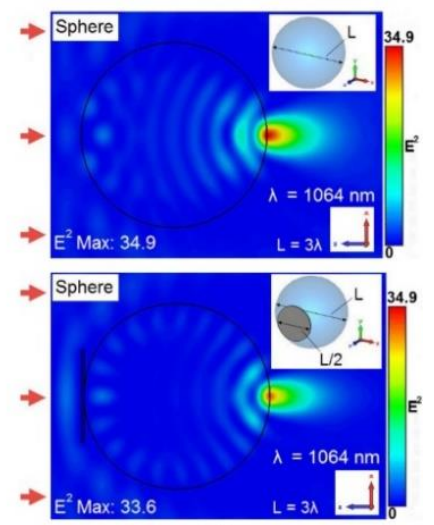

c)

Fig.4(i). $E^{2}$ field enhancement distribution in $x z$ plane for the $3 \lambda$ particle-lenses dimensions in the shapes of the axially illuminated circular-column (a), cubic (b), and spherical particle (c) without/with the mask apodization.

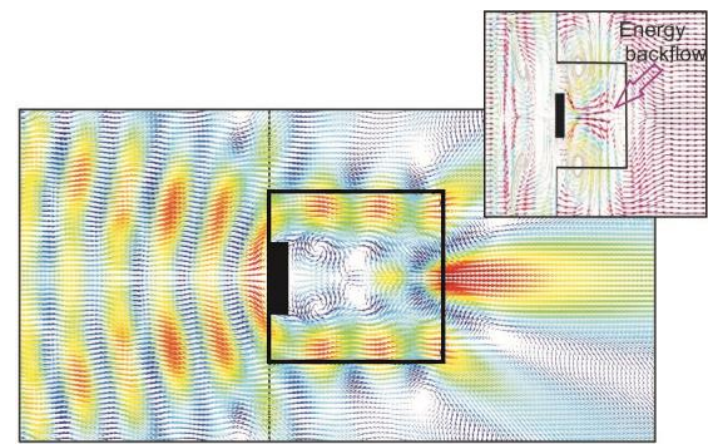

Fig.4(ii). Distribution of the Poiting vector within the optimized cubic structure. The inset shows the vortex structure of the field, which blocks the formation of a photonic jet.

The location of the mask, its material and geometric dimensions make it possible to optimize the vortex structure (optical flow) of the field inside the dielectric particle and, thereby, to control the parameters of the photonic jet (Fig.4(ii)). On the other hand, the organization of the vortex configuration of the field near the shadow surface of the particle makes it possible to block the formation of the photonic jet. In particular, it opens up prospects (see insert in Fig.4(ii)) to study the bound state in the continuum due to strong coupling between FabryPerot-like and Mie-like modes, and optical backflow in such structures.

\section{Tangential electric field component control}

Due to the contrast of the refractive index, the incident electromagnetic wave is delayed inside the particle. But it is also continuously delayed near the outer surface of the particle due to the continuity of the tangential components of the electric field, which results in a concave curvature of the wavefront near the focus. Thus the photonic jet parameter control is possible due to the control for the tangential electric field component along the lateral surface of the dielectric particle using, for example, a metal mask placed along its lateral surfaces $[52,53]$. With metal screens placed along the lateral surface of the dielectric particle, a reflected wave occurs, since the respective field component disappears within the contact area. After the wave reflection, the amplitude of the tangential electric field component grows in the direction opposite to that of the wave propagation, and then weakens in the 
wave propagation direction [52]. These effects lead to a shift of the power flow density concentration region both crosswise and in the wave propagation direction [54]. In particular, it becomes possible to "lock" the field localization region inside a dielectric particle with a refractive index less than the critical one when the screen moves along the lateral surface of this particle. We called this effect the "optical syringe". This allows controlling the focal distance of the photonic jet, its length along the radiation propagation direction, and reducing the beam waist near the focus area. On the other hand, the presence of a screen near one lateral particle surface provides the formation of photonic hooks and angular steering by a photonic jet [53]. Note that the role of the tangential field component is important from a practical point of view, such as, for example, fixing a particle in a microscope holder [55], explaining the effect of increasing focal length.

\section{Terajets}

The term terajet is introduced by Minin et al. [56] in relation to a photonic jet in the microwave and/or terahertz range. The main difference from optics is that in the terahertz range, the absorption coefficient of the particle material plays a significant role. It can exceed the optical range by several orders of magnitude, and the particle almost always cannot be considered as a weakly absorbed. Note that the first experimental data on the photonic jet effect are presented in [57] for the microwave range.

Dielectric cubic particles have certain advantages over spherical particles [26]: at a linear polarization, the photonic jet generated by the sphere is more elliptical than that generated by cubic particles; with increasing size of cuboid and the sphere diameter, the jet length grows for cuboids and shortens for spheres; cuboids generate photonic jets with the minimum particle size of 0.5 wavelength, while spherical - with the minimum diameter of more than a wavelength, etc.

According to [56], the terajet ("teraknife" in a two-dimensional case) moves from inside to outside of the structure with decreasing refractive index of three- and two-dimensional cuboids. It provides a subwavelength size of the beam waist and a high intensity, which is 10 times higher than the intensity of the illuminating wave. The properties of the dielectric cubic particle as an analog of a flat lens having sizes of the order of a wavelength, are retained at the plane wave inclination both on the fundamental frequency and frequency harmonics [58]. Moreover, on a par with the optical range [19], the possibility of the backscattering enhancement by $\sim 1.53$ and $\sim 10 \mathrm{~dB}$ was demonstrated for metal particles $d_{1}=0.1 \lambda$ and $d_{2}=0.25 \lambda$ in a terajet field, respectively [56].

The use of metamaterials for mesoscale particles allows one to observe new effects, for example, birefringence of the photonic jet [26]. In [26] the results for the photonic jet formation based on 2D photonic crystals (PhC) with high index dielectric cylindrical rods (n $=3.32$ ) in air host medium are discussed. It has been shown that high-intensity peaks are localized in a small volume of the PhC within rods, which cannot be predicted from the Bloch wave's theory. Taking into account that the length of a photonic jet for TE-mode is about 2 times less than that for TM-mode, the photonic jet is birenfringent. In [59] two other different types of metamaterial heterogeneous structures based on photopolymerperforated cuboid were considered: rectangle with circle holes in homogenious dielectric 
and alternating layered structure. The smallest beam waist (almost $0.2 \lambda$ ) has been observed for TM-polarized wave in photopolymer PLA. The results shown that it is possible to separate in space photonic jets for the TE- and TM-polarized waves [26].

The interesting terajet applications include the gain in sensitivity of terahertz detectors by localized downward radiation in a subwavelength volume [60-62], gain in sensitivity of open microwave resonators [63] (see Fig.5a), the use of terajet generating mesoscale particles as a dielectric small-sized antenna in promising terahertz communication systems, including 5G-6G [64, 65], (see Fig.5b), and others.

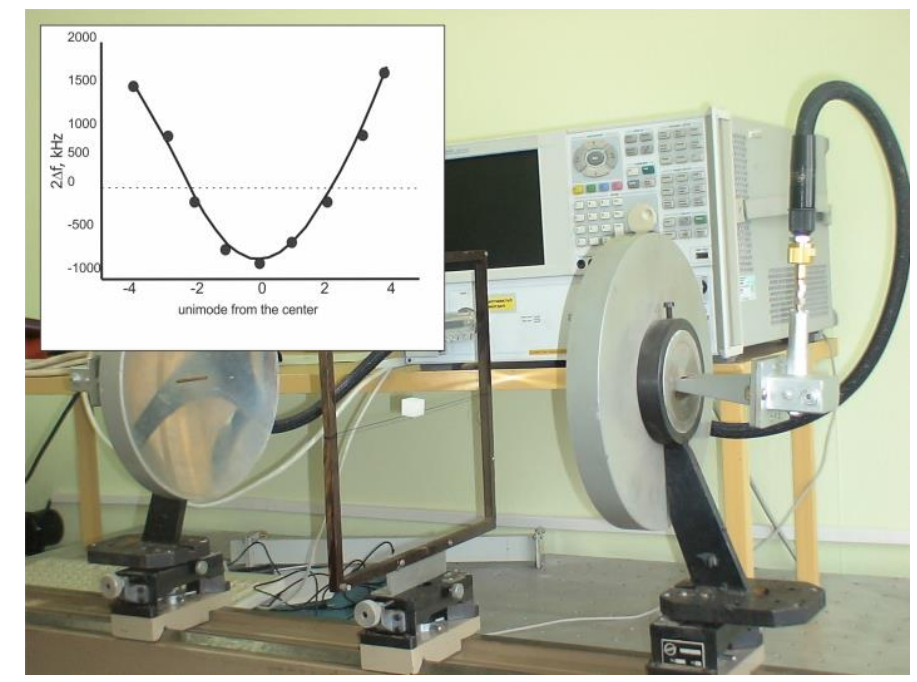

Fig.5a. The $2 \Delta f$ dependencies $5.53 \mathrm{GHz}$ mode [63] from the shift of dielectric balls with diameter of 16 $\mathrm{mm}$ to the sides in the antinodes of the electric field along the resonator axis.

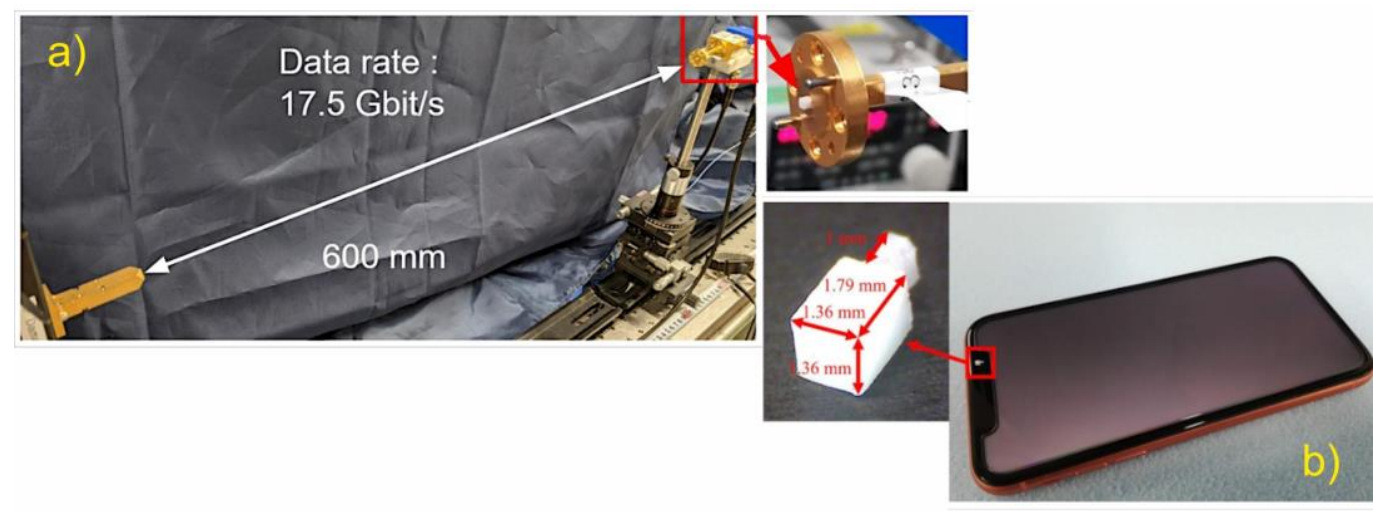

Fig.5b. THz communication experiment at $300 \mathrm{GHz}$ (a) and artist view of dielectric mesoscale cuboid antenna implementing in mobile phone (b). Adapted from [64,65].

Placing a mesoparticle in the antinode of an open resonator (Fig.5a) increases the Q-factor of the resonator, since the terajet decreases the size of the antinode in the center of the resonator, providing an extremely low effective mode volume, which leads to a decrease in diffraction losses at the edges of the mirrors. We refer the reader to [63] for the detailed discussion. 


\section{Anomalous Gouy phase shift}

The additional information about phase fields can remove restrictions to the problem of super-resolution imaging. The Gouy phase shift [66] was investigated for spherical particles with size parameters $q=9.34$ [67] and $q=21.4$ [68]. It is shown that the phase shift along the axis of the photonic jet propagation, is a combination of the classical Gouy phase shift and the phase shift induced by a refraction on a spherical particle.

At the same time, for cubic particles with the size parameters of $q=3$ (the cube side equals the wavelength), the Gouy phase shift model based on the focused Gaussian beam, cannot explain the Gouy phase shift in the photonic jet [69]. Therefore, the formation mechanism of the terajet-based (photonic jet) localized radiation differs from that of the classical lens or spherical particle.

Moreover, in [70] it was reported on the Gouy asymmetric phase anomaly in a terajet under oblique illumination of a dielectric cube. This anomaly produced the angle of deviation, not known before, between the normal of the Gouy phase distribution and the direction of the incident beam propagation in the place of the photonic jet formation. This angle of deviation exponentially reduced with the wave propagation to a distance of several wavelengths.

The understanding of such phase anomalies allows localizing the radiation in spatial regions that are smaller than the classical diffraction limit of resolution and can be applied in microscopy, optical trapping and nanoparticle manipulations, interferometry [71].

\section{Waveguide systems based on dielectric particles chain}

Goubau and Sobel developed the first prototype of the lens waveguide with a set of identical lenses in 1961 [72, 73]. In optics, the light periodic focusing effects were studied in relation to a chain of weakly absorbing spheres $2 \mu \mathrm{m}$ in size $(q>12)$ and the refractive index higher than 1.3. It was shown that these effects were attributed to the so-called photonic modes induced by photonic jets with a period of about a size of two spheres arranged close to each other [74-76]. Interestingly, due to photonic modes induced by photonic jets, when using a linearly polarized Gaussian pulse of femtosecond duration, for a chain of spherical particles with the same size parameter $\mathrm{q}$, temporal broadening of the pulse at a distance of about 4 particle diameters is not observed. However, in the case of propagation of the same pulse in a solid cylinder made of the same material and diameter, the temporal broadening of the pulse is approximately two times.

In our work [77], we propose another type of the waveguide consisting of a chain of threedimensional mesoscale cubic particles (with the side equaling to the wavelength) separated by an air gap (quasi-periodic dielectric waveguide). The work demonstrates the existence of protonic periodic focusing modes induced by terajets along the particle chain. Also, we show that the proposed structure possesses reliable performance and is lowsensitive to the particle material losses (up to the tangent losses of $\sim 0.1$, which is $5 \ldots 6$ orders higher than in optics) due to the fact, that the spatial resolution of terajets does not considerably lower near the shadow-side surface of each cube at a significant loss increase. Unlike the optical waveguide based on spherical particles, the waveguide based on cubic particles does not require the microsphere contact with each other. The contact between 
spherical particles can be implemented by microwelding, which, however, decreases the waveguide properties due to microcracks forming after the material melting in the contact area $[78,79]$.

The light periodic focusing effects are also observed for a chain of mesoscale particles with $n \sim 1$ refractive index [80]. As can be seen from Fig. 6, we have a waveguide with the graded index, i.e., the refractive index gradually lowers from the waveguide center to its edge due to a specific particle shape (sphere, cube) at a constant refractive index inside the particles. Although the downward radiation propagates at the same rate at the waveguide input, the time of reaching its output is different, since the wave propagation velocity at the end is higher than at the center, which leads to the modal dispersion. In fact, this type of the waveguide can be considered as an analog of the waveguide with the gradient (parabolic) refractive index $[81,82]$.

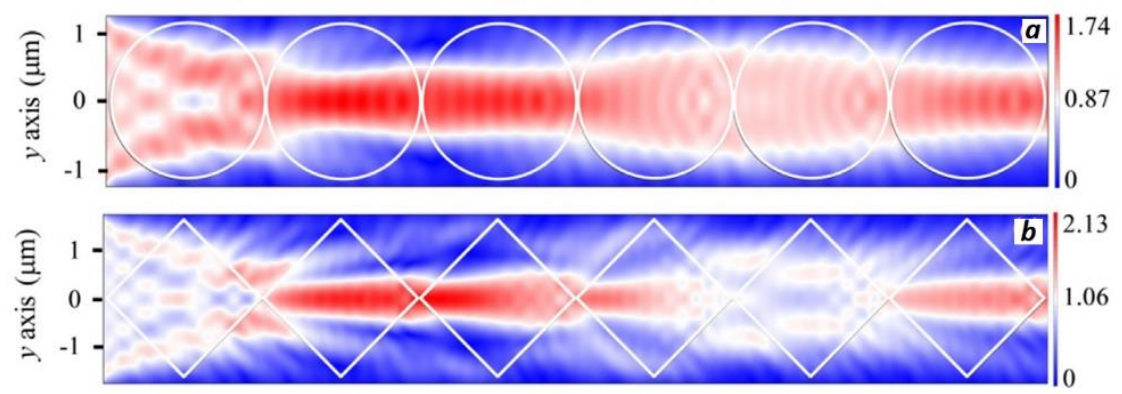

Fig. 6. Waveguide with $(a)$ spherical $(n=0.87)$ and $(b)$ cubic $(n=1.06)$ particle chains.

It is interesting to mention the use of mesoscale particles alloyed by optically nonlinear and/or active materials that provides rather interesting optical parametric and laser effects in these structures can be used to improve the resolution of an isolated particle in the farfield region.

\section{Specular-reflection photonic nanojet}

A specular-reflection photonic nanojet is a type of electromagnetic subwavelength spatial radiation localized in the near-field region, which results from constructive interference of direct and back waves focused by a transparent dielectric mesoscale particle locating near the flat reflecting mirror, and is directed toward the wavefront incident $[27,28]$.

For example, the thickness selection of the dielectric particle locating on the metal screen provides the formation of the photonic jet with a 1.07 ellipticity, i.e., almost axially symmetric focus at linear polarization. The control for the spatial position of the photonic jet is also possible, until it becomes parallel to the flat surface of the metal screen. This control is performed through a rotation of the whole structure relative to the radiation incidence $[27,83]$. Such a way of the structured field formation is possible for dielectric mesoscale particles of different shapes, including hemispherical [84].

The applicability of Babinet's principle of the additional diffraction structures also demonstrated [85] for the photonic jet generation in reflection mode in the near-field region. Such structures open up possibilities of creating photonic jets with required and controlled properties, such as focal distance, width, length, maximum field strength, and jet ellipticity. 
At the same time, the photonic jet formation in the reflected wave allows avoiding several restrictions to the refractive index of the particle material, associated with the area of the photonic jet existence in the propagated radiation [5]. According to [86], the photonic jet can form in the opposite direction of the incident wave propagation, in using a near-unityrefractive-index particle on a dielectric substrate with high index contrast or a metal substrate. In this case, the beam width can less than the diffraction limit. The analysis of the Poynting vector distribution shows that this phenomenon is associated with a multiple circulation of the power flux in and near the particle. It should be noted that the selection of the refractive index contrast between the particle and the substrate, can provide the parameter control of the localized radiation area and its position in both reflected and propagated waves. In Fig. 7, one can see the electric field intensity distribution at the incident plane wave on the spherical particle with $\sim 1(n=1.077)$ refractive index placed on dielectric substrates with 3.83 and 4.08 refractive indices, respectively. In the first case, we observe a standing wave-modulated photonic jet in the reflection mode and a weak region of localization in the propagated wave. And in the second case, we see the refractive focusing in the propagated wave and a weak localization in the reflected wave.

Another unique property of photonic jets in the reflection mode, is their spatial subwavelength localization and high intensity in using mesoscale particles with a relatively high contrast of the refractive index, when a common photonic jet does not generate $(n>2)$ [87]. In such a configuration, a double focusing of the plane incident wave occurs with the help of a particle locating near the flat metal mirror.

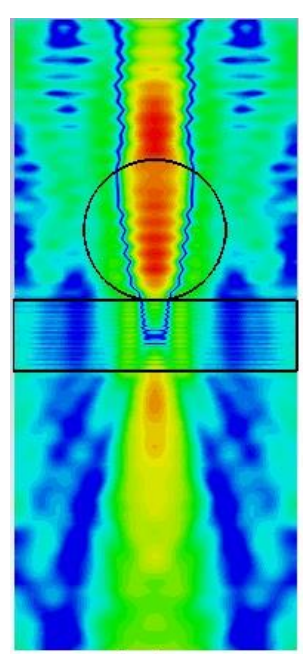

(a)

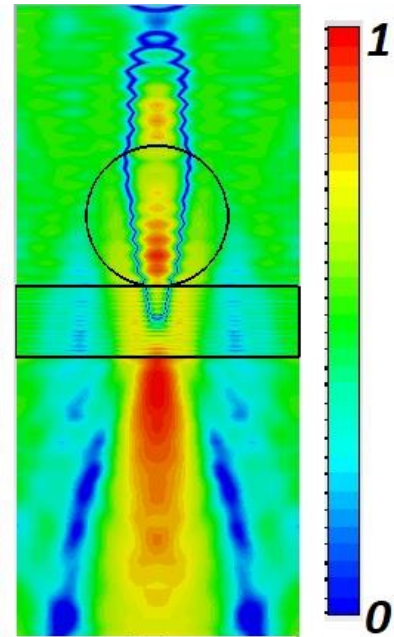

(b)

Fig. 7. Generation of photonic jet for spherical particle $(n=1.077)$ positioned on dielectric substates: a) $\left.n_{1}=3.83, b\right) n_{1}=4.08$.

This possibility to overcome the fundamental restriction of the maximum allowable contrast of the refractive index of the particle material is conditioned by the fact that at a specular reflection of radiation followed by the dielectric-particle focusing, large-scale vortices with oncoming circulating optical energy appear in this particle, that contributes to the extreme value of the optical field concentration.

In [87] it was proposed a physical concept of the optical tweezer, in which the stability to the Brownian motion of a trapped particle was two times higher than in conventional 
trappings based on photonic nanojets [88]. The standing wave modulation of the photonic jet could be eliminated, if necessary, due to the interference control [89].

The property control of the spatial propagation of narrow light beams such as divergence, focusing, are the main problems in optics and photonics. Another interesting photonic jet effect is that the dielectric-metal thin rectangular structure can function as a flat focusing mirror $[27,28,90]$. The new type of a planar device without the pre-specified optical axis [90] provides focusing in the near-field region, where the structured reflected beam demonstrates non-diffracted propagation by a distance of the photonic jet length and can significantly enhance the applicability of structured photonic systems to control the light beam propagation in mesoscale photonic circuits. Note that this type of the near field focusing flat mirror is much simpler than that proposed in [91,92] with a transverse invariance, and is based on other physical principles.

\section{Overcoming the diffraction limit and image quality improvement}

According to [93], the effects of whispering gallery mode in microsphere leads to super-resolution imaging up to $\sim 0.25 \lambda$. In [7], it is shown that at a resonance frequency matching the size parameter $q=26.94163$ of the spherical particle, the hotspot size can be about $0.22 \times 0.37 \lambda$ for the linear polarization of the incident field at over 43000 intensity. The achieved focusing parameters are very sensitive to the size parameter of the spherical particle, i.e., its change by $10^{-4}$ reduces the intensity by 4 orders of magnitude. The similar effect of resonance super-resolution imaging was considered in [94]. It is interesting to note that $0.27 \times 0.6 \lambda$ focal spot for the linear polarization of the illuminating wave can be obtained in an off-resonance frequency mode for a specific configuration of a zone plate [95] with a low level of the side lobes, but at a maximum field intensity, which 1000 times lower. This is shown in Fig. 8.
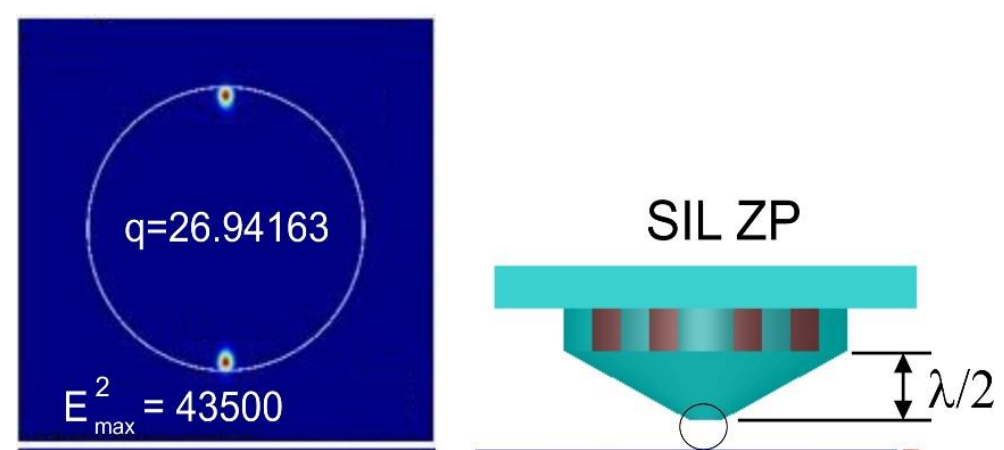

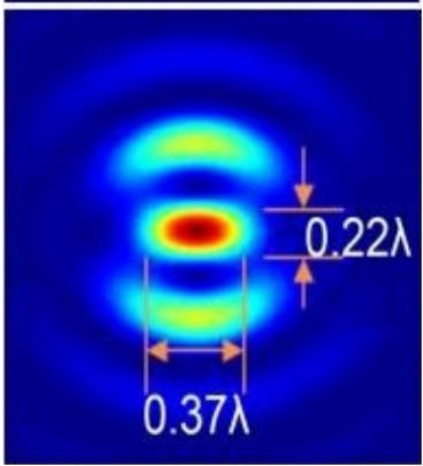

(a)

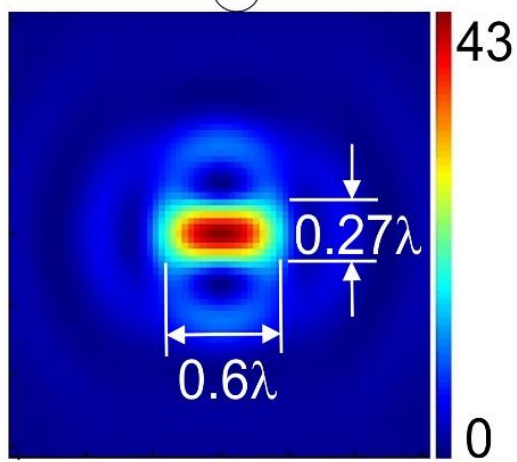

(b)

Fig. 8. Formation of localized radiation for spherical particle at resonance frequency (a) and zone plate with immersion cone on off-resonance frequency (b) at linear polarization. 
The hotspot generation effect at the poles of spherical particles can be used to detect nanoparticles, including viruses, when their presence in the hotspot region must lead to the resonance frequency shift.

A transfer from common to metamaterials allows dielectric particles with a size of about a wavelength to gain super-resolution, i.e., the beam waist size up to 0.2 wavelength [96], which is rather smaller than the classical diffraction limit. At the same time, the reduction in the beam waist size is also possible due to the use of the gradient particle materials [97]. These effects can assist in solving problems of optical metrology of super-high resolution [98].

In [99] we study a new mechanism of the field localization in mesoscale Janus particles [100] based on truncated spheres or cylinders. It is found that the electric field on a plane surface of such particles has sharp resonances depending on the depth of the removed segment of a sphere or cylinder. These resonances are attributed to the excited whispering gallery modes caused by a truncation of the particle surface. This effect optimized for cylindrical particles allows reaching super-resolution on their plane surface [99].

The physical principles of the truncated spherical lens with focusing without aberrations, also known as the Weierstrass lens, are based on the beam compression formed by the decrease in the refracting angle of the propagated radiation measured on the optical axis. This occurs, when the sphere is truncated down to thickness of $d=R^{*}(l+l / n)$, where $R$ is the sphere radius, $d$ is the applanate focus [101, 102]. But for low truncation values (small $d$ values), the field redistribution is observed due to strong excitation of whispering gallery modes and the enhancement of both electric and magnetic fields. Singularity associated with the phase discontinuity on a trace of spherical (cylindrical) surface and plane surface changes Snell's law into laws of reflection and refraction [103]. In accordance with this law, the critical angles of the total internal reflection change, and a critical angle of incidence exists at some value of phase gradient [103]. The latter depends on the thickness of the truncated $d$ element and the refractive index $n$. Figure 9 presents the interference of two evanescent waves. It is found that the effectiveness of excitation by the whispering gallery wave method strongly depends on the truncated $d$ element.
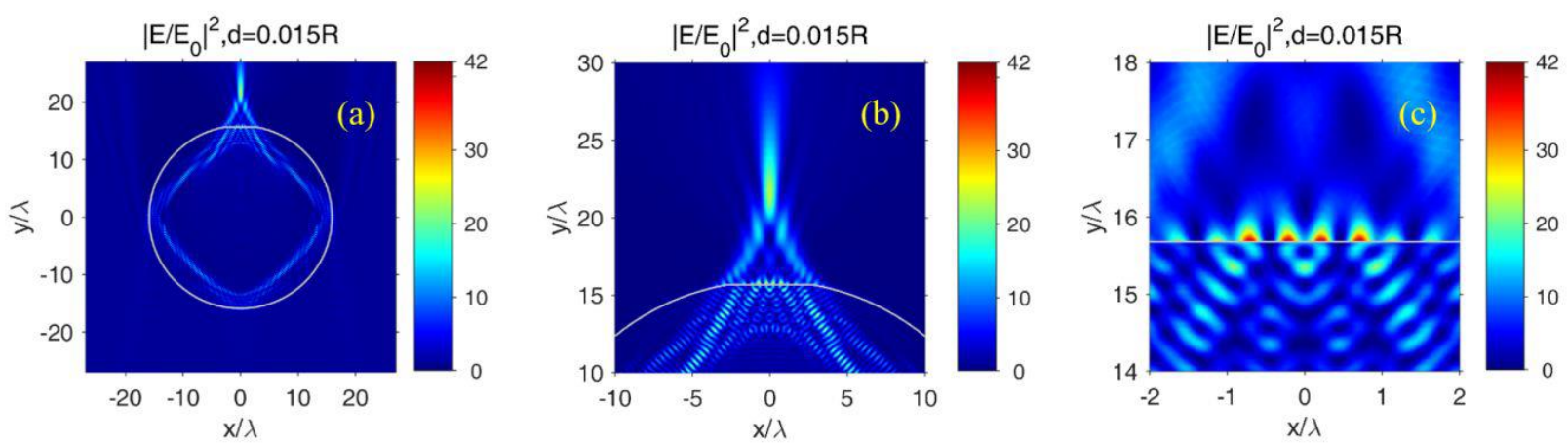

Fig. 9. Field intensity distribution for truncated segment resonance and its fragments. Plane front incidents from bottom to top. Adapted from [99]. 
The use of Janus particles provides the growth in robustness and electric and magnetic field components near the surface of the removed particle element. This effect is resonance in relation to the volume of the removed fraction of the substance and is observed within $q=30 \ldots 90$.

The microsphere introduction in the circuit of a common optical microscope leads to undesired aberrations that lower the image contrast and quality despite the improved localized resolution in the region under the microsphere. Minin et al. [104] demonstrate for the first time that a Teflon dielectric cube with the side equaling a wavelength, can be used to improve the spatial resolution of terahertz imaging systems by its placing in the focal area of a lens, independently of the numerical aperture. This approach can be used both in reflecting and transmitting imaging systems [105]. In using $125 \mathrm{GHz}$ frequency in image formation systems, the resolution can be enhanced by 2.2 times, which matches $275 \mathrm{GHz}$. This is especially important in the terahertz range, where the frequency growth of the probing radiation leads to the increase in the medium absorption, and thus cannot be applied. In this case, the image contrast enhancement is 4.4 .

The lowest resolution in optical microscopy is limited by the fundamental diffraction limit. Microscopes based on dielectric microparticles are promising tools for overcoming the diffraction limit. But microparticles have a low image contrast in air, which restricts the application of this method. Using the microparticles that provide the formation of the localized radiation at an angle of the radiation incidence (optical axis), it is possible to increase the image contrast, for example, by photonic hook or jet with oblique subwavelength illumination in near field [106]. By analogy with classical optical microscopes [107, 108], in the case of the axial formation of the photonic jet, the radiation falls onto an object, and diffracted orders of -1 and +1 are beyond the particle boundaries, while oblique illumination results in the diffracted order of +1 on the particle, which, finally allows increasing the image contrast [109] (see Fig. 10).
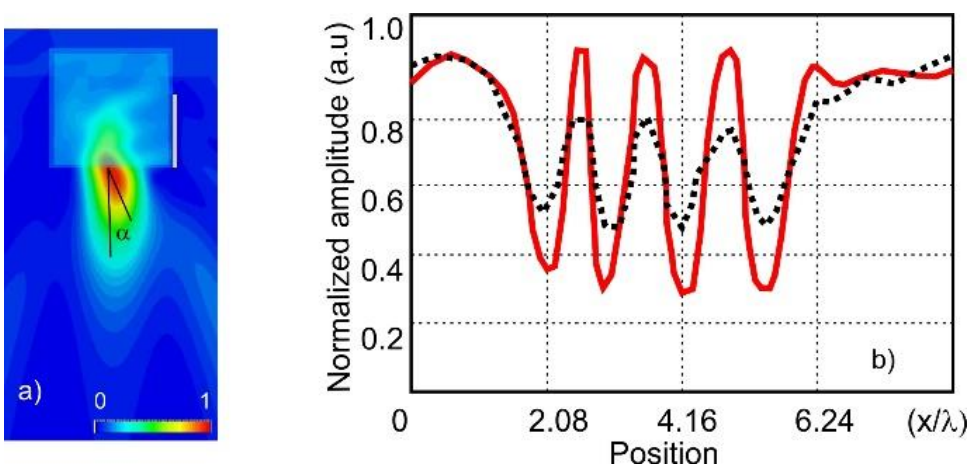

Fig. 10. Formation of photonic hook for cubic particle wit lateral mask (a); image contrast of test object in photonic jet radiation (dotted curve) and photonic let (solid curve) (b).

\section{Nanostructured particles with refractive index near 2}

As mentioned above, the transverse size of the localized field of a dielectric mesoscale particle is usually larger than $\lambda / 3$ at the refractive index below the critical. For the first time, in $[110,111]$, it is shown that the size of the region of localized electromagnetic field 
forming near the shadow surface of the dielectric mesoscale spherical or cubic particle with a refractive index of 2 of its material, can be substantially reduced after the introduction of nanoholes on its shadow surface, which improves the spatial resolution up to $\lambda / 100$. Such an introduction allows compressing the field localization characteristic to the photonic jet, down to the size of this nanohole thanks to the permittivity contrast between the particle and nanohole; the minimum beam waist is determined by the nanohole size rather than the wavelength. At the same time, an analysis of the optical response of a particle based on the multipole decomposition showed that in the nonresonant mode the nanohole weakly affects the optical response of the particle.

It is known that a circulating energy flow arises around a phase singularity containing a reverse flow [2-5]. Thus, the localized field region formed by a mesoscale particle with the critical or slightly higher than the critical refractive index contrast [87, 112] and having a nano-hole on the axis, can be used to separate regions with forward and backward energy flows [113]. Such nanostructuring of the shadow-side surface of the particles with the refractive index 2 provides the development of the concept of the optical vacuum cleaner [111] and optical magnet [113] for manipulation of nanoparticles, and also an optical modulation based on wavelength scaled dielectric particle with graphene monolayer [112]. A more complex nanostructuring of the shadow-side surface of the particles with the refractive index below 2 , based on the diffraction structures for optical manipulation was considered in [114].

\section{Structured fields in the form of photonic hooks and loops}

Recently, a new family of near-field localized curved light beams was discovered, known as photon hooks, which differ from Airy beams $[115,116]$. The term photonic hook and its concept were introduced in $[117,26]$. Photonic hooks are unique, because their curvature radius is much smaller than the wavelength, which means that such hooks can be maximally accelerated, which the side lobes do not fit the shape of the main beam and are not curved. Moreover, in photonic hooks, there is a flex point, where the beam changes the direction of propagation. Photonic hooks can be observed near the shadow-side surface of the Janus particles, and it can be free form other restrictions. Airy beams have no such properties [116].

In the simplest case, Janus particles in the form of a dielectric cube with a prism [117, 26, $118,119]$, interference induced by the different particle thickness along the polarization direction, lead to the additional singular points near the shadow-side surface of the asymmetric particle. In the latter, the time of the total phase of the optical wave oscillation changes, resulting in the beam curvature. In other words, it is known that the photonic energy is able to flow in and out through nano-vortexes representing the singularities of Poynting vectors associated with topological charge. Consequently, the layout of these vortexes influence the flow direction and intensity of Poynting vectors, especially in the areas which are close to the particle boundaries. The asymmetric vortexes of Poynting vector streamlines in the asymmetric dielectric particle lead to the formation of a curvilinear localized beam near the shadow surface of the particle. Moreover, a change in 
the wavelength or/and polarization of the incident radiation leads to a spatial redistribution of nanovortices, as a result of which the position and structure of the photonic hook in space changes.

There are currently several approaches to the formation of the curved beams such as photonic hooks, systemized in [120]. The approaches include asymmetry of the particle shape at a constant refractive index [121, 122], symmetric particles with refractive index asymmetry [123-125], symmetric uniform particle with asymmetric illuminating wave [126, 127], symmetric particles in inhomogeneous (e.g., with discrete refractive index distribution) medium, a system o mesoscale scatters [128], and a combination of these approaches. These approaches can generate localized structured beams of various configuration. In Fig. 11(i), one can see the field configurations for a photonic hook and optical capsule, and an optical trapping based on a structured cylindrical particle (cylinder with $\pi / 2$-cut segment). In the latter case, the cylindrical particle is irradiated by a spatiallimited wave, whereas the cut segment in the shadow-side surface provides the formation of two photonic jets, whose interference forms the region of a standing wave of higher intensity (a photonic jet from the upper half of the cylinder falls on the flat surface of the cut segment and is reflected from it). As shown in Fig. 11(ii), the elongated shape of the bottle-like region of such 'nanophotonic tweezers,' an exciting new class of 'on-chip' optical traps, provides the use of such a trapping for anisotropic nanoobjects that is rather difficult in classical configurations. These concepts also can be developed to obtain nearfield beams of the photonic loop type [125].
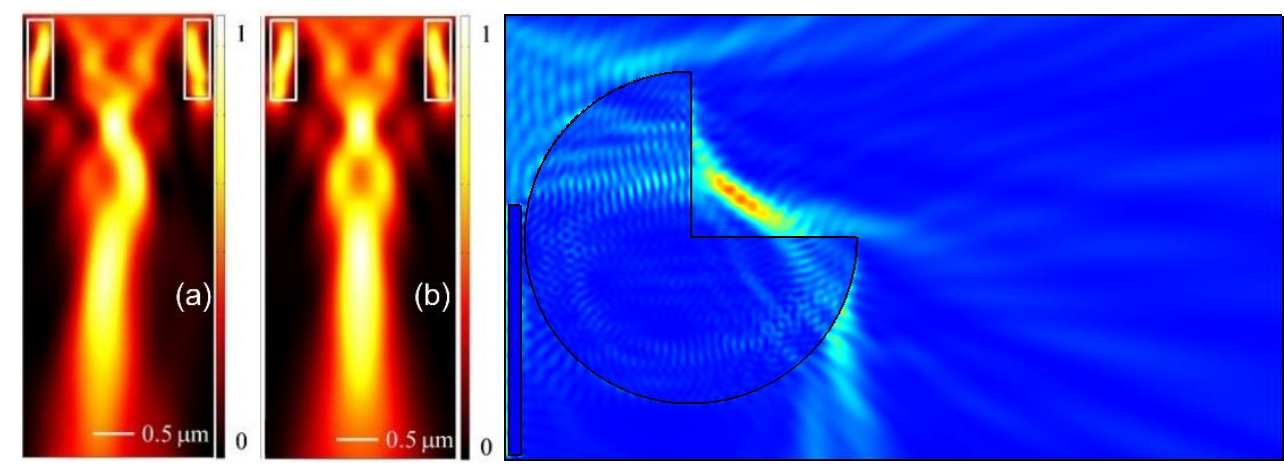

Fig. 11(i). Structured fields in the form of photonic hook (a) and bottle-like region of localized field (b) based on a pair of dielectric rods. Right: optical trapping in standing wave region.

Structured light in step-index media like photonic hook is shown in Fig.11d (The simulation Comsol model was created by Yu.Geints). In this case, the mesoparticle is located in a homogeneous medium (in this example, in air), but in the shadow part of the particle there is an inhomogeneous medium, for example, a two-layer step-index air-water structure. The choice of the thickness of the layers of water and air determines the configuration of the structured localized field. In this case, a significant role in the formation of a structured field is played by the position of the interface between the waterair layers, since a vortex flow is formed inside the particle in the contact region of these 
two media (power flow is shown in the inset). Note that due to the gradient of the refractive index contrast of the two media, the localized beam modulated by a standing wave.

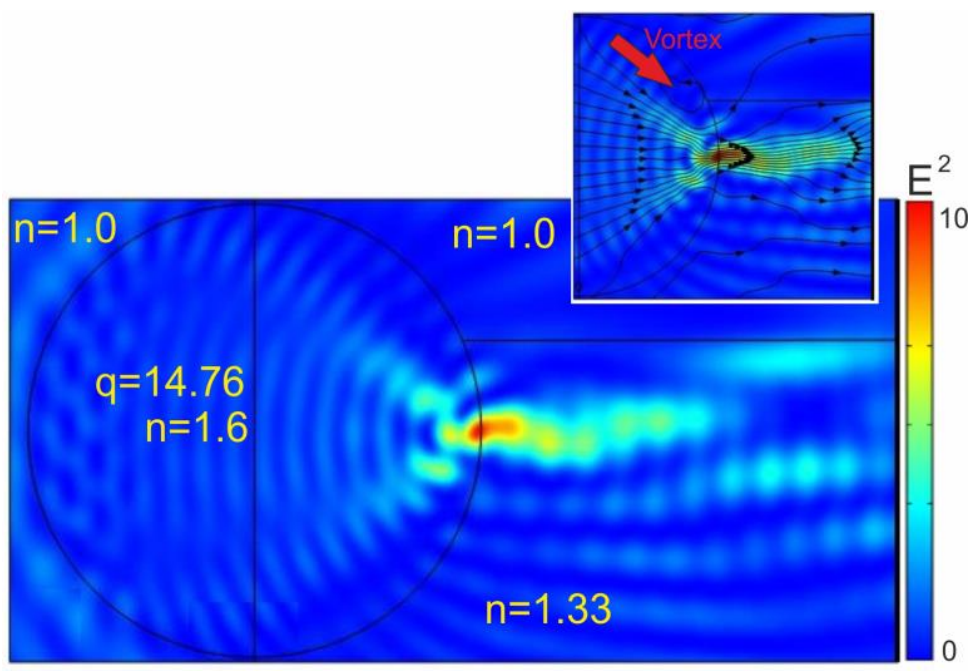

Fig. 11(ii). Concept of the structured light in the form of photonic hook in step-index media

The photonic hook concept suggests the control for the particle motion to manipulate and sort cells on the laboratory on-a-chip platform. A unique property of the photonic hook is the subwavelength beam curvature, that can be used to transport nanoparticles outside the obstacle [117]. In the approximation of a Rayleigh particle, it is shown that it can be moved round the dielectric plate-obstacle [129]. At the same time, a simple change in polarization can switch the spatial orientation of the region of field localization [126, 127]. Some of in vitro biomedical applications of this concept include the cell motion over the curved trajectory for their successive differentiation [130], and nanoobject transportation to the intercellular or intracellular media by a curved trajectory for nanodrug delivery via the central nervous system barriers [131], laser surgery [132], microfluid devices, surface nanostructuring, new photonic devices [133], and many others.

The near-field control will be more and more important in the integrated photonics. For example, prospects are opening up for constructing devices for spectral and spatial separation of radiation incident on a particle, using the phenomenon of a photonic hook and a jet. In this regard, structured beams of the photonic jet and photonic hook type, can be a bridge to connect the photonic and physical worlds. It is interesting to note that the properties of pyramidal meso-particles [27, 28, 134, 135], probably, disclose the nature of ancient pyramids [136-138].

\section{Low-dimensional systems. Plasmonic jets and hooks}

The study of the properties of low-dimensional systems occupies one of the central positions in photonics. Plasmonic structures are intensively studied due to their ability to localize light at subwavelength scales $[139,140]$. Surface plasmonic waves are two- 
dimensional waves, whose field components exponentially decay as they move away from the metal-dielectric interface.

Fundamentally, in the transition to two-dimensional space from three-dimensional, the quantum nature of matter manifests itself. Therefore, there are new possibilities for controlling the interaction between material and light. The possibility of formation of the photonic jet analog for plasmonic waves based on a dielectric disk placed on a metal film was first shown theoretically in [141]. Later, based on the solution of Maxwell's equations, the formation of a plasmonic jet based on a dielectric cuboid of silicon nitride $\left(\mathrm{Si}_{3} \mathrm{~N}_{4}\right)$ was investigated in [142]. Using the idea of a waveguide based on the cubic particle chains [77], the possibility of increasing the propagation length of the localized plasmonic wave was demonstrated in [143].

However, the plasmonic jet effect based on a dielectric cube on a gold film was first confirmed experimentally in [144]. The plasmonic jet effect demonstrated exciting potential applications in integrated and near-field optics for the plasmonic wave control. Such a new and simple platform could provide a new path for plasmonics, high-resolution imaging, biophotonics, optical data storage, and future applications from nanoscale light control to on-chip photonic devices [145].

The same relates to the plasmonic hook. The plasmonic hook effect was theoretically justified in [146], and just recently it was first confirmed experimentally [147]. The singular points inside the Janus particle induce the curvatures of the plasmonic beam. It is important to note that the plasmonic hook demonstrated the smallest beam curvature ever recorded for plasmonic waves. It could be noted that using the idea of a surface plasmon linear waveguide [143] and results of the works [146,147] it is possible to construct curved surface plasmon waveguides using an array of Janus particles arranged along a curved path.

The experimental verification of these effects opens up new possibilities for controlling the interaction between the light and matter at the nano-level and could greatly expand the available tools for new light manipulations [148].

\section{Acoustic jets and hooks}

In the optical range, two parameters are usually sufficient to characterize photonic jets: the Mie size parameter and the refractive index contrast. In the terahertz and microwave ranges, a parameter characterizing losses in the particle material is added. In the acoustic range, another parameter is added; all materials, as a rule, are anisotropic due to the presence of two sound velocities in the solid particle material: longitudinal and transverse (shear wave).

The term acoustic jet was introduced by Minin I.V. and Minin O.V. in [149] by analogy with Maxwell's equations and continuum mechanics for the linear mode. Works [150, 151] experimentally demonstrated the acoustic jet generation with a minimum beam waist size of the acoustic jet of about half the wavelength for spherical and cylindrical particles. A significant influence on the formation of the acoustic jet exerted by the ratio between the axial and transverse sound velocities in the particle material was shown in [152]. Acoustic 
jets based on metamaterial particles were investigated in [149, 153]. The effect of anomalous apodization for acoustic jets in both gas and liquid was confirmed in [154, 155]. It could be noted that a more detailed study showed that a simple analogy between acoustics and optics [149] is valid only in the first approximation, since it is necessary in acoustics to use one more additional free parameter - the particle density [120].

According to Fig. 12, in the acoustic jet formation, just as in optics, an essential role is given to the system of acoustic vortices both inside the particle material and near its shadow surface.
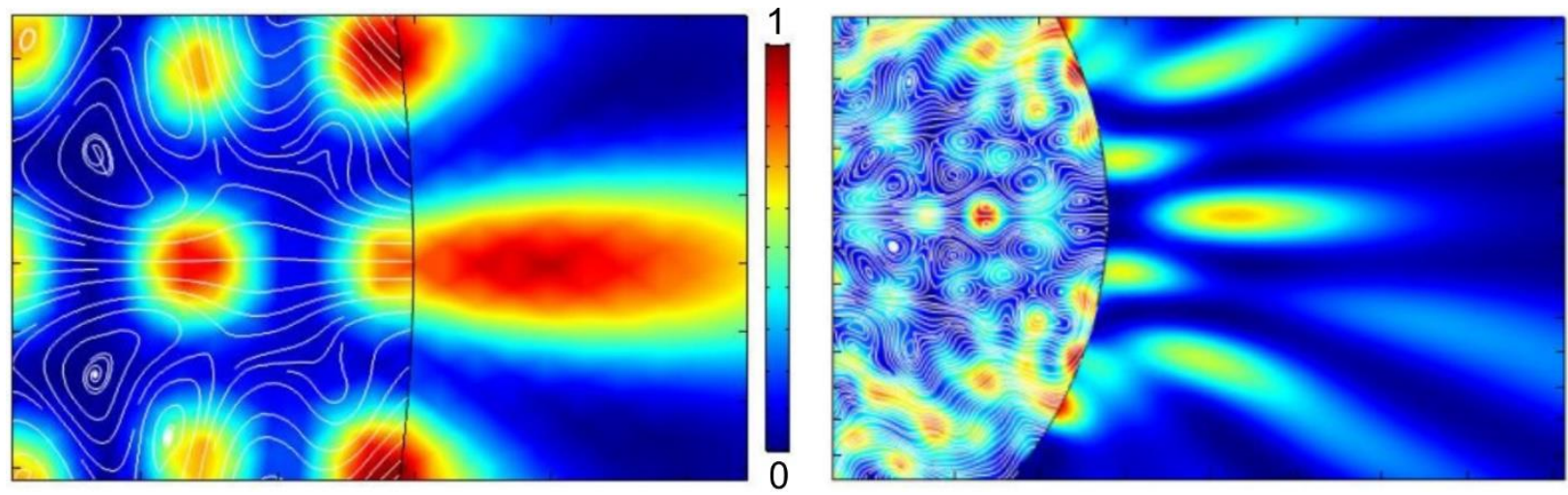

Fig. 12. Vortices in the particle material (Rexolite density: $1049 \mathrm{~kg} / \mathrm{m}^{3}$, axial sound velocity: $2337 \mathrm{~m} / \mathrm{s}$, transverse sound velocity: $1157 \mathrm{~m} / \mathrm{s}$ ) and hotspot generation in off-resonance (left) and near-resonance (right) modes.

In addition, the optical super-resonance effect were also observed in acoustics [156, 157] as well as extremely high acoustic wave intensity (see Fig. 13). Near the resonance frequency, the resolution in the hotspot region is about $(0.2-0.23) \lambda$.

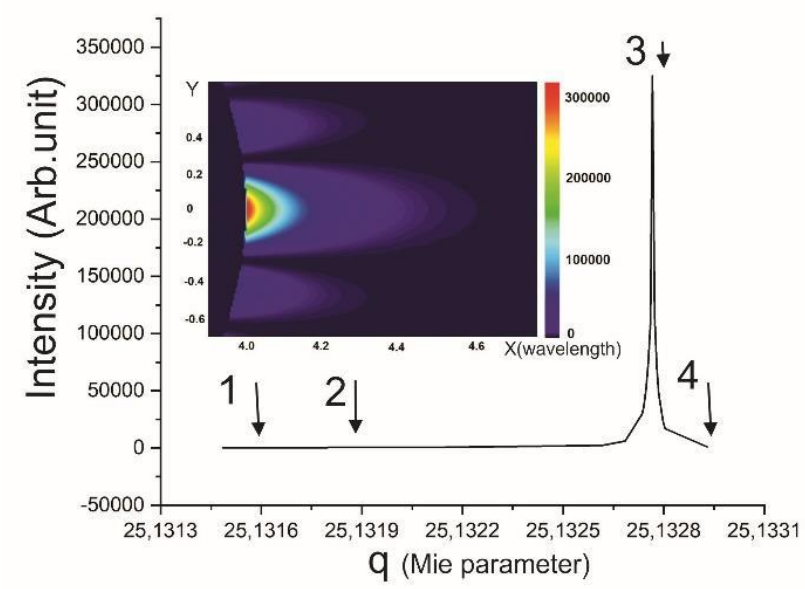

Fig. 13. Resonance scattering of acoustic wave on a spherical Rexolite particle located in water depending on Mie parameter. Inset: hotspot structure on the particle shadow-side surface at the resonant frequency. Maximum intensity at points 1-4: 325 (1), 2500 (2), 325000 (3), 1000 (4) in units of the acoustic wave intensity incident on the particle.

The photonic hook concept was extended to acoustic waves and experimentally confirmed in [158]. This curved acoustic beam was generated by using a Rexolite particle immersed 
in water with a trapezoidal rectangular prism, on which a plane ultrasonic wave with a $250 \mathrm{kHz}$ frequency fell. The same results were obtained later in [159]. In [160], acoustic jets and hooks in water were obtained using a mesoscale $(q<30)$ ABS plastic cylinder fabricated on a $3 \mathrm{D}$ printer. It was shown that in the resonance mode, the excitation of whispering gallery modes resulted in the acoustic jet diameters less than the diffraction limit. In the off-resonance mode, a cubic phononic crystal was placed inside the cylinder, and both acoustic jets and acoustic hooks were formed depending on its orientation relative to the wave incidence direction. Later, a particle consisting of a hemispherical Rexolite cylinder and a hemispherical photonic crystal (two different materials with different effective refractive indexes were used) forming an acoustic hook was considered in [161].

The structured acoustic beams such as jets and hooks can also find applications in microscopy, medical imaging [162], monitoring of various structural states [163], energy harvesting and localization [164].

\section{Conclusions}

The electromagnetic field localization and manipulation in the small volume up to a deep subwavelength, are fundamental for research into dielectric mesophotonics. Main attention in this paper was paid to novel strategies employed to manipulate subwavelength structured optical, acoustic and in-plane plasmonic fields, enhance localized magnetic and electric fields, and photonic wavelength-scaled particle-based waveguide.

The field of dielectric 2D- and 3D- mesostructures is a relative new emerged research field in photonics and in-plane plasmonics (and even in acoustics) that is based on the physics of relative low-index dielectric wavelength-scaled particles with Mie size parameter of q 10 empowered by interference effects between various fields components generated in or/and near the shadow surface of those particles. In this case, different possibilities are provided both in non-resonant and resonant modes. An additional key point is that dielectric mesoscale particles can be fabricated out of well-known dielectric materials with simple shape, for example, sphere, cylinder or cubic, that can naturally be made using standard processing. On the other hand one of the limitation of mesoparticles is the fact that field localization is limited to particle sizes of the order of $2 R>\lambda$.

Mesoparticles may support high-order Fano super-resonances, which make it possible to implement giant magnetic field generation as well as hot spots generated by high-order Fano resonances can be apply to superresolution microscopy.

Optical singularities, in particular vortices and their acoustic counterparts, are intriguing deep physics phenomena that allow for interesting applications. For example, the angular and linear momentum of photons in structured fields can be used for optical capture and manipulation of nanoparticles and biological micro-objects $[165,166]$.

With auxiliary mesoscale dielectric particles of an arbitrary 3D shape, it was possible to generate structured fields, which allowed increasing the flexibility and creating more varied kinds of optical manipulation with unusual properties of the optical forces beyond trapping. 
The generation of giant electric and magnetic fields in dielectric mesoscale particles is comparable to the fields generated in plasmonic structures and can be used to destroy viruses [167], optical capture of particles with a high value of magnetic polarizability [168]. The idea of the photothermal therapy method proposed in [169], is to deliver a heat dose via metal nanoparticles, lethal for cancer cells, to a given area of living tissue. These nanoparticles are introduced into the body and bind to cancers. But the traditional gold spherical nanoparticles have an absorption peak in the visible range. In order to shift the plasmon resonance toward the near-infrared range and trap nanoparticles by the target, MXene $\mathrm{Ti}_{3} \mathrm{C}_{2}$ nanoparticles in the field of the photonic hook are used in [122].

A separate major direction is associated with the use of mesoscale particles in microscopy $[38,39,104,170]$. Also a promising way from our point of view is the way of creating assembled mesoparticles with the required properties based on phase-matching mechanism that relies on the coupling of random quasi-phase-matching with the Mie resonances of the entire disordered mesostructure [171]. The generation of hot spots, having giant values of the local wavenumber vectors, by use a superoscillation effects $[9,10]$ extremely promising not only for mesoscale photonics and superresolution imaging, but also for diffractive optics [172], whose research was begun back in 1990 [173].

The use of Janus particles, the simplest of which is a hemisphere (half-cylinder) [174], with various types of asymmetry mentioned above, allows for flexible control of structured fields depending on the application. The particle in resonant fundamental mode (modes with the smallest volume corresponding to $|\mathrm{m}|=1$, and $\mathrm{s}=1$ ) allow to use it as a cloak structure [175].

These results could be useful for research into trapping, control, and detection of natural, biological, and artificial nanoparticles, including molecules and viruses. At present, interest in topics briefly discussed above is growing noticeably, which can be explained by both its fundamental and applied aspects.

We believe new insights being delivered by merging the concepts from several adjacent fields and we expect that the future research in dielectric mesophotonics will offer new perspectives, leading to novel unusual applications for photonics. It seems that the combination of the concept of mesoscale particles with materials with nonlinear properties will open up new and attractive perspectives. The results reveal the potential of wavelength-scaled dielectric particles to manipulate radiation beyond the limitations of geometrical macro optics [176] (effects negligible at the macro optics become important at the mesoscale, and vice versa) and their potential for various different applications remains to be investigated. In general, the number of new physical effects and applications indicates to the development of a new promising direction in optics, acoustics, and in-plane plasmonics.

Author Contributions: Conceptualization, I.M. and O.M.; methodology, I.M. and O.M.; writingoriginal draft preparation, I.M. and O.M. All authors have read and agreed to the published version of the manuscript.

Acknowledgments: The authors are grateful to all of co-authors who contributed directly to published joint papers in different subject. The work was carried out within the framework of the Tomsk Polytechnic University Development Program, Russia.

Conflicts of Interest: The authors declare no conflict of interest. 


\section{References}

1. Mie, G. Contributions to the optics of turbid media, particularly of colloidal metal solutions. Ann. Phys. 1908, 25(3), 377445.

2. Luk'yanchuk, B.; Ternovsky, V. Light scattering by a thin wire with a surface-plasmon resonance: Bifurcations of the Poynting vector field. Phys. Rev. B 2006, 73, 235432.

3. Bashevoy, M.; Fedotov, V. and Zheludev, N. Optical whirlpool on an absorbing metallic nanoparticle. Opt. Express 2005, 13(21), 8372.

4. Luk'yanchuk, B.; Miroshnichenko, A. and Kivshar, Y. Fano resonances and topological optics: an interplay of far- and near-field interference phenomena. J.Opt. 2013, 15, 073001.

5. Luk'yanchuk, B.; Paniagua-Dominguez, R.; Minin, I.V.; Minin, O.V.; Wang, Z. Refractive index less than two: Photonic nanojets yesterday, today and tomorrow, Optical Materials Express, 2017, 7(6), 1820 - 1847.

6. Yue, L.; Yan, B.; Monks, J.; Dhama, R.; Jiang, C.; Minin, O. V.; Minin, I.V. \& Wang, Z. Full three-dimensional Poynting vector flow analysis of great field-intensity enhancement in specifically sized spherical-particles. Sci. Rep. 2019, 9, 20224.

7. Wang, Z.; Luk'yanchuk, B.; Yue, L.; Yan, B.; Monks, J.; Dhama, R.; Minin, O. V.; Minin, I.V.; Huang, S. \& Fedyanin, A. High order Fano resonances and giant magnetic fields in dielectric microspheres. Sci Rep 2019, 9, 20293.

8. Yue, L.; Wang, Z.; Yan, B.; Monks, J.; Joya, Y.; Dhama, R.; Minin, O.V. and Minin, I.V. Super-Enhancement Focusing of Teflon Spheres. Ann. Phys. (Berlin) 2020, 532, 2000373.

9. Berry, M. V. Superoscillations and leaky spectra. J. Phys. A Math. Theor. 2018, 52, 015202.

10. Zheludev, N. I. \& Yuan, G. H. Optical superoscillation technologies beyond the diffraction limit. Nat Rev Phys (2021). https://doi.org/10.1038/s42254-021-00382-7.

11. Sakharov, A. D. Magnetoimplosive generators. Soviet Physics Uspekhi. 1966, 9(2), 294-299.

12. Minin, V.F.; Minin, I.V. and Minin, O.V. Calculation Experiment Technology. In: Computational Fluid Dynamics. InTechOpen (2011). DOI: 10.5772/22497

13. Chen, Z.; Taflove, A. and Backman, V. Photonic nanojet enhancement of backscattering of light by nanoparticles: a potential novel visible-light ultramicroscopy technique. Opt. Express 2004, 12, 1214-1220.

14. Benincasa, D. S., Barber, P. W., Zhang, Jian-Zhi, Hsieh, Wen-Feng, \& Chang R. K. Spatial distribution of the internal and near-field intensities of large cylindrical and spherical scatterers. Applied Optics, 1987, 26(7), 1348-1356.

15. Luk'yanchuk, B.; Zheng, Y. \& Lu, Y. Laser cleaning of solid surface: Optical resonance and near-field effects, Proc. SPIE $4065,2000,576-587$.

16. Lu, Y.; Zhang, L.; Song, W.; Zheng, Y. \& Luk'yanchuk, B. Laser writing of a subwavelength structure on silicon (100) surfaces with particle-enhanced optical irradiation. Jetp Lett. 2000, 72, 457-459.

17. Mosbacher, M.; Münzer, H.; Zimmermann, J.; Solis, J.; Boneberg, J. \& Leiderer, P. Optical field enhancement effects in laser-assisted particle removal, Appl. Phys. A 2001, 72, 41-44.

18. Wang, J.; Gouesbet, G.; Gréhan, G.; Han, Y. and Saengkaew, S. Morphology-dependent resonances in an eccentrically layered sphere illuminated by a tightly focused off-axis Gaussian beam: parallel and perpendicular beam incidence. J. Opt. Soc. Am. A, 2011, 28(9), 1849.

19. Li, X., Chen, Z., Taflove, A., \& Backman, V. Optical analysis of nanoparticles via enhanced backscattering facilitated by 3D photonic nanojets. Opt. Express, 2005, 13, 526.

20. Yousefi, M.; Scharf, T. and Rossi, M. Photonic nanojet generation under converging and diverging beams. J. Opt. Soc. Am. B 2021, 38(2) 317-326. 
21. Wang, H.; Hao, J.; Zhang, B.; Han, C.; Zhao, C.; Shen, Z.; Xu, J. and Ding, J. Donut-like photonic nanojet with reverse energy flow. Chinese Optics Letters 2021, 19(10), 102602.

22. Lecler, C.; Takakura, Y.; Meyrueis, P. Properties of a three-dimensional photonic jet. Opt.Lett. 2005, 30, 2641-2643.

23. Minin, I.V.; Minin, O.V.; Gagnon, N. and Petosa, A. FDTD Analysis of a Flat Diffractive Optics with Sub-Reyleigh Limit Resolution in MM/THz Waveband," 2006 Joint 31st International Conference on Infrared Millimeter Waves and 14th International Conference on Teraherz Electronics, 2006, 170-170.

24. Minin, I.V. and Minin, O.V. Experimental verification 3D subwavelength resolution beyond the diffraction limit with zone plate in millimeter wave. Microwave and Optical Technology Letters, 2014, 56(10), 2436-2439.

25. Liu, C.; Kaiser, T.; Lange, S. and Schweiger, G. Structural resonances in a dielectric sphere illuminated by an evanescent wave, Opt. Commun. 1995, 117, 521-531.

26. Minin, I.V. and Minin, O.V. Diffractive Optics and Nanophotonics: Resolution Below the Diffraction Limit, Springer, Berlin, Germany 2016.

27. Minin, I.V. and Minin, O.V. Photonics of isolated dielectric particles of arbitrary 3D shape - a new direction of optical information technologies. Vestnik NSU 2014, 12(4), 59-70.

28. Minin, I.V.; Minin, O.V.; Geints, Y. Localized EM and photonic jets from non-spherical and non-symmetrical dielectric mesoscale objects: Brief review. Ann. Phys. (Berlin). 2015, 527(7-8), 491-502.

29. Cui, X.; Erni, D.; Hafner, C. Optical forces on metallic nanoparticles induced by a photonic nanojet. Opt. Express, 2008, 16(18), 13560.

30. Li, Y.; Xin, H.; Liu, X.; Zhang, Y.; Lei, H.; Li, B. Trapping and Detection of Nanoparticles and Cells Using a Parallel Photonic Nanojet Array, ACS Nano 2016, 10(6), 5800.

31. Shakhov, A.; Astafiev, A.; and Nadtochenko, V. Microparticle manipulation using femtosecond photonic nanojet-assisted laser cavitation, Optics Letters 2018, 43(8), 1858.

32. Surdo, S.; Duocastella, M.; and Diaspro, A. Nanopatterning with Photonic Nanojets: Review and Perspectives in Biomedical Research, Micromachines 2021, 12(3), 256.

33. Lu, D.; Pedroni, M.; Labrador-Paez, L.; Marques, M.; Jaque, D. and Haro-Gonzalez, P. Nanojet Trapping of a Single Sub-10 nm Upconverting Nanoparticle in the Full Liquid Water Temperature Range, Nano Micro Small 2021, 17(7), 2006764.

34. Killian, J.; Ye, F. and Wang, M. Optical Tweezers: A Force to Be Reckoned With, Cell 2018, 175(6), $1445-1448$.

35. Shan, X.; Wang, F.; Wang, D.; Wen, S.; Chen, C.; Di, X.; Nie, P.; Liao, J.; Liu, Y.; Ding, L.; Reece, P. and Jin, D. Optical tweezers beyond refractive index mismatch using highly doped upconversion nanoparticles, Nature Nanotechnology 2021, $16,531-537$.

36. Zhang, W. and Lei, H. Fluorescence enhancement based on cooperative effects of a photonic nanojet and plasmon resonance, Nanoscale 2020, 12(12), 6596-6602.

37. Bratchenko, L.; Bratchenko, I.; Khristoforova, Y.; Artemyev, D.; Konovalova, D.; Lebedev, P. and Zakharov, V. Raman spectroscopy of human skin for kidney failure detection, Journal of Biophotonics 2020, 14(6), e202000360.

38. Hüser, L. and Lehmann, P. Microsphere-assisted interference microscopy for resolution enhancement tm - Technisches Messen, 2021, 88(5), 311-318. https://doi.org/10.1515/teme-2020-0101

39. Li, P.; Li, G.; Yu, H.; Wang, F.; Liu, L. and Li, W. Advances in Dielectric Microspherical Lens Nanoscopy: Label-Free Superresolution Imaging. IEEE Nanotechnology Magazine, 2021, 15(1), 38.

40. Kuznetsov, A.; Miroshnichenko, A.; Fu, Y.; Zhang, J. \& Luk'yanchuk, B. Magnetic light. Scientific Reports $2012,2,492$.

41. Albella, P.; Poyli, M.; Schmidt, M.; Maier, S.; Moreno, F.; Saénz, J. and Aizpurua, J. Low-Loss Electric and Magnetic FieldEnhanced Spectroscopy with Subwavelength Silicon Dimers, J. Phys. Chem. C 2013, 117(26), 13573-13584. 
42. Bakker, R.; Permyakov, D.; Yu, Y.; Markovich, D.; Paniagua-Domínguez, R.; Gonzaga, L.; Samusev, A.; Kivshar, Y.; Luk'yanchuk, B. and Kuznetsov, A. Magnetic and electric hotspots with silicon nanodimers, Nano Lett. 2015, 15(3), 21372142.

43. Li, C.; Kattawar, G. and Zhai, P. Electric and magnetic energy density distributions inside and outside dielectric particles illuminated by a plane electromagnetic wave, Opt. Express 2005, 13(12), 4554.

44. Baranov, D.; Savelev, R.; Li, S.; Krasnok, A. and Alu, A. Modifying magnetic dipole spontaneous emission with nanophotonic structures, Laser Photonics Rev. 2017, 1600268.

45. Born, M. and Wolf, E. Principles of Optics 7th edn (Cambridge: Cambridge University Press), 1999

46. Yan, B.; Yue, L.; Wang, Z. Engineering near-field focusing of a microsphere lens with pupil masks. Opt. Commun. 2016, 370, 140.

47. Wu, M.; Chen, R.; Soh, J.; Shen, Y.; Jiao, L.; Wu, J.; Chen, X.; Ji, R. \& Hong, M. Super-focusing of center-covered engineered microsphere. Sci Rep. 2016, 6, 31637.

48. Minin, I. V. and Minin, O. V. Patent of Russia 2015, 21, N 153686.

49. Yue, L.; Yan, B.; Monks, J.; Dhama, R.; Wang, Z.; Minin, O.V. and Minin, I.V. Intensity-Enhanced Apodization Effect on an Axially Illuminated Circular-Column Particle-Lens. Ann. Phys. (Berlin) 2017, 1700384

50. Yue, L.; Yan, B.; Monks, J.; Wang, Z.; Tung, N.; Lam, V.; Minin, O.V. and Minin, I.V. Production of photonic nanojets by using pupil-masked 3D dielectric cuboid. J. Phys. D: Appl. Phys. 2017, 50, 175102.

51. Yue, L.; Yan, B.; Monks, J.; Wang, Z.; Tung, N.; Lam, V.; Minin, O.V. and Minin, I.V. A millimetre-wave cuboid solid immersion lens with intensity-enhanced amplitude mask apodization. J Infrared Milli Terahz Waves, 2018, 39(6), 546-552.

52. Liu, C.; Chen, W.; Geints, Y.; Minin, O.V. and Minin, I.V. Simulation and experimental observations of axial position control of photonic nanojet by dielectric cube with metal screen. Optics Letters 2021, 46(17) 4292-4295.

53. Liu, C.; Chen, W.; Minin, O.V. and Minin, I.V. Multispectral photonic jet shaping and steering by control of tangential electric field component on cuboid particle. Photonics 2021, 8, 317

54. Dorofeev, I.; Suslyaev, V.; Minin, O.V. and Minin, I.V. Role of the tangential electric field component to the terahertz jet and hook formation by dielectric cube and sphere. Opt. Eng. 2021, 60, 082004.

55. Ling, J.; Li, D.; Liu, X.; Wang, X. Ultra-long focusing of microsphere lens via wavefront reconstruction in microsphere, Proc. SPIE 10964, 2018, 109642D.

56. Pacheco-Pena V., Beruete M., Minin I. V., Minin O. V. Terajets produced by 3D dielectric cuboids, Appl. Phys. Lett., 2014, $105,084102$.

57. Zhao L. and Ong C. K. Direct observation of photonic jets and corresponding backscattering enhancement at microwave frequencies. J. Appl. Phys., 2009, 105, 123512.

58. Pacheco-Pena V., Beruete M., Minin I. V., Minin O. V. Multifrequency focusing and wide angular scanning of Terajets. Optic Letters, 2015, 40(2), 245.

59. Khodzitsky, M.; Vozianova, A.; Gill, V.; Chernyadiev, A.; Grebenchukov, A.; Minin, I.V. and Minin, O.V. Formation of terahertz beams produced by artificial dielectric periodical structures, Proc. SPIE 9918, 2016, 99182X.

60. Minin, I. V., O. V. Minin, J. Delgado-Notario, et al., Improvement of a terahertz detector performance using the terajet effect in a mesoscale dielectric cube: Proof of concept, Physica Status Solidi-Rapid Research Letters, 2020, 14(5), 1900700.

61. Minin, I. V., O. V. Minin, J. Salvador-Sanchez, et al., Responsivity enhancement of a strained silicon field effect transistor detector at $0.3 \mathrm{THz}$ using the terajet effect, Optics Letters, 2021, 46(13), 3061-3064.

62. Minin, O. V., I. V. Minin, Y. M. Meziani, et al., Improvement of a point-contact detector performance using the terajet effect initiated by photonics, Opt. Eng. 2020, 60(8), 082005. 
63. Dorofeev, I.; Dunaevsky, G.; Suslyaev, V.; Minin, I.V. and Minin, O.V. Small-sized body influence on the quality factor increasing of quasioptical resonator. Optical and Quantum Electronics 2017, 49, 355-10

64. Samura, Y., K. Horio, V. B. Antipov, et al., Characterization of mesoscopic dielectric cuboid antenna at millimeter-wave band, IEEE Antennas and Wireless Propagation Letters, 2019, 18(9), 1828-1832.

65. Yamada, K.; Samura, Y.; Minin, O.V.; Kanno, A.; Sekine, N.; Nakajima, J.; Minin, I.V; Hisatake, S. Short-range Wireless Transmission in the 300-GHz Band Using Low-profile Wavelength-scaled Dielectric Cuboid Antennas. Front. Comms. Net 2021, 2, 702968 .

66. Gouy, L. G. Sur une propriete nouvelle des ondes lumineuses. C. R. Acad. Sci. Paris 1890, 110, 1251.

67. Kim, M.; Scharf, T.; Mühlig, S.; Rockstuhl, C. and Herzig, H. Gouy phase anomaly in photonic nanojets. Appl. Phys. Lett. 2011, 98, 191114.

68. Bon, P.; Rolly, B.; Bonod, N.; Wenger, J.; Stout, B.; Monneret, S. and Rigneault, H. Imaging the Gouy phase shift in photonic jets with a wavefront sensor, Opt. Lett. 2012, 37, 3531-3533.

69. Pham, H.; Hisatake, S.; Minin, I.V.; Minin, O.V. and Nagatsuma, T. Three-dimensional direct observation of Gouy phase shift in a terajet produced by a dielectric cuboid. Appl. Phys. Lett. 2016, 108, 191102.

70. Pham, H.; Hisatake, S.; Minin, O.V. Nagatsuma, T. and Minin, I.V. Asymmetric phase anomaly of terajet generated from dielectric cube under oblique illumination. Appl. Phys. Lett. 2017, 110, 201105.

71. Liñares, J.; Prieto-Blanco, X.; Montero-Orille, C. and Moreno, N. Spatial mode multiplexing/demultiplexing by Gouy phase interferometry, Opt. Lett. 2017, 42, 93-96.

72. Christian, G. and Goubau, J. Experimental studies on a beam waveguide for millimeter waves. IRE Trans. Antennas Propag. $1961,9,256$.

73. Sobel, F.; Wentworth, F. and Wiltse, J. Quasi-Optical Surface Waveguide and Other Components for the 100-to 300-Gc Region. IRE Trans. Microwave Theory Tech. 1961, 9, 512.

74. Chen, Z.; Taflove, A. and Backman, V. Highly efficient optical coupling and transport phenomena in chains of dielectric microspheres, Opt. Lett. 2006, 31, 389-391.

75. Kapitonov, A. and Astratov, V. Observation of nanojet-induced modes with small propagation losses in chains of coupled spherical cavities, Opt. Lett. 2007, 32, 409-411.

76. Yang, S. and Astratov, V. Photonic nanojet-induced modes in chains of size-disordered microspheres with an attenuation of only $0.08 \mathrm{~dB}$ per sphere, Appl. Phys. Lett. 2008, 92, 261111.

77. Minin, I.V.; Minin, O.V.; Pacheco-Peña, V. and Beruete, M. All-dielectric periodic terajet waveguide using an array of coupled cuboids. Appl. Phys. Lett. 2015, 106, 254102.

78. Mitsui, T.; Wakayama, Y.; Onodera, T.; Hayashi, T.; Ikeda, N.; Sugimoto, Y.; Takamasu, T. and Oikawa, H. Microdemultiplexer of Coupled Resonator Optical Waveguide Fabricated by Microspheres, Adv. Mater., 2010, 22, $3022-3026$.

79. Mitsui, T.; Onodera, Y.; Wakayama, T.; Hayashi, N.; Ikeda, Y.; Sugimoto, T.; Takamasu, H.; Oikawa, H. Influence of microjoints formed between spheres in coupled resonator optical waveguide, Opt. Express 2011, 19, 22258-22267.

80. Liu, C.; Minin, O. V. and Minin, I.V. Periodical focusing mode achieved through a chain of mesoscale dielectric particles with a refractive index near unity. Optics Communications 2019, 434, 110-117.

81. Mikaelyan, A. SELFOC dielectric waveguides, Sov. J. Quantum Electron. 1977, 7, 266-267.

82. Liu, L.; Rahman, M.; Ning, Y. and Grattan, K. Accurate mode characterization of graded-index multimode fibers for the application of mode-noise analysis, Appl. Opt. 1995, 34, 1540-1543.

83. Minin, I.V.; Minin, O.V.; Pacheco-Peña, V. and Beruete, M. Localized photonic jets from flat, three-dimensional dielectric cuboids in the reflection mode, Opt. Lett. 2015, 40, 2329-2332. 
84. Minin, I.V.; Minin, O.V.; Kharitoshin, N. Localized High Field Enhancements from Hemispherical 3D Mesoscale Dielectric Particles in the Reflection Mode. Proc. of the 16th Int. Conf. EDM, 29 June-3 July 2015, Novosibirsk, Russia, 2015, $331-333$.

85. Minin, I.V.; Minin, O.V.; Nesedov, I. Photonic jets from Babinet's cuboid structures in the reflection mode, Opt. Lett. 2016, $41,785-787$.

86. Yue, L.; Yan, B.; Monks, J.; Dhama, R.; Wang, Z.; Minin, O.V. and Minin, I.V. Photonic Jet by a Near-Unity-RefractiveIndex Sphere on a Dielectric Substrate with High Index Contrast. Ann. Phys. (Berlin) 2018, 1800032.

87. Minin, I.V.; Geints, Y.; Zemlyanov, A. and Minin, O.V. Specular-reflection photonic nanojet: physical basis and optical trapping application. Optics Express 2020, 28(15), 22690.

88. Wang, H.; Wu, X. and Shen, D. Trapping and manipulating nanoparticles in photonic nanojets, Optics Letters 2016, 41(7), 1652.

89. Sergeeva, K.; Sergeev, A.; Minin, O.V. and Minin, I.V. A Closer Look at Photonic Nanojets in Reflection Mode: Control of Standing Wave Modulation. Photonics 2021, 8, 54.

90. Minin, I.V.; Liu, C.; Yang, Y; Staliunas, K. \& Minin, O.V. Experimental observation of flat focusing mirror based on photonic jet effect. Sci Rep 2020, 10, 8459.

91. Cheng, Y., Kicas, S., Trull, J., M. Peckus, C. Cojocaru, R. Vilaseca, R. Drazdys \& K. Staliunas. Flat Focusing Mirror. Sci Rep 2014, 4, 6326.

92. Cheng, Y. and Staliunas, K. Near-field flat focusing mirrors. Applied Physics Reviews 2018, 5, 011101.

93. Zhou, S.; Deng, Y.; Zhou, W.; Yu, M.; Urbach, H.; Wu, Y. Effects of whispering gallery mode in microsphere super-resolution imaging. Appl. Phys. B 2017, 123, 236.

94. Hoang, T.; Duan, Y.; Chen, X.; Barbastathis, G. Focusing and imaging in microsphere-based microscopy. Opt. Express 2015, 23, 12337-12353.

95. Geints, Y.; Panina, E.; Minin, I.V. and Minin, O.V. Study of focusing parameters of wavelength-scale binary phase Fresnel zone plate. J. Opt. 2021, 23, 065101.

96. Minin, I. V. and O. V. Minin, Terahertz artificial dielectric cuboid lens on substrate for super-resolution images, Opt. and Quant. Electron., 2017, 49(10), 326.

97. Liu, C.; Yen, T.; Minin, O. V. and Minin, I.V. Engineering photonic nanojet by a graded-index micro-cuboid. Physica, E. Low-dimensional systems \& nanostructures, 2018, 98, 105-110.

98. Kwon, S.; Kim, K.; Park, J.; Cho, Y.; Lee, M. Microsphere-assisted ultra-small spot spectral reflectometry technique for semiconductor device metrology, Proc. SPIE 11611, 2021, 116110G.

99. Minin, I. V.; Minin, O.V.; Cao, Y.; Yan, B.; Wang, Z.; Luk'yanchuk, B. Photonic Lenses with whisphering Gallery Waves at Janus Particles. Opto-Electronic Science 2022, accepted. ArXiv: 2012.09489 (2020).

100. de Gennes, P.-G. Soft Matter (Nobel Lecture). Angewandte Chemie International Edition in English. 1992, 31(7), $842-845$.

101. Mason, D.; Jouravlev, M.; Kim, S. Enhanced resolution beyond the Abbe diffraction limit with wavelength-scale solid immersion lenses, Optics Letters 2010, 35, 2007-2009.

102. Kino, G. and Mansfield, S. Near field and solid immersion optical microscope. US Patent 5,004,307, 1991.

103. Yu, N.; Genevet, P.; Kats, M.; Aieta, F.; Tetienne, J.; Capasso, F.; Gaburro, Z. Light Propagation with Phase Discontinuities: Generalized Laws of Reflection and Refraction. Science 2011, 334, 333-337.

104. Pham, H.; Hisatake, S.; Minin, O.V.; Nagatsuma, T. and Minin, I.V. Enhancement of Spatial Resolution of Terahertz Imaging Systems Based on Terajet Generation by Dielectric Cube APL Photonics 2017, 2, 056106.

105. Minin, I. V.; Minin, O.V.; Salvador-Sanchez, J.; Delgado-Notario, J.; Calvo-Gallego, J.; Baranov, P.; Ferrando-Bataller, M.; Fobelets, K.; Velazquez-Perez, J. and Meziani, Y. Imaging resolution enhancement using terajet effect at 0.3THz. Proc. SPIE $11868,2021,118680 \mathrm{~N}$. 
106. Minin I.V., Minin O.V. Terahertz Microscopy with Oblique Subwavelength Illumination in near Field. Preprints 2021090024 (2021); doi: 10.20944/preprints202109.0024.v1

107. Abbe, E., On the estimation of the aperture of the microscope, J.Roy Microscop. Soc. Ser. II 1881, 1, 349-388.

108. Sanchez, C., Cristobal, G., Bueno, G., Blanco, S., Borrego-Ramos, M., Olenici, A., Pedraza, A., Ruiz-Santaquiteria, J. Oblique illumination in microscopy: a quantitative evaluation. Micron 2018, 105, 47-54.

109. Minin, I.V. and Minin, O.V. THz microscopy with oblique subwavelength illumination: proof of concept. Quantum Electronics, 2022 (accepted)

110. Cao, Y.; Liu, Z.; Minin, O.V. and Minin, I.V. Deep Subwavelength-Scale Light Focusing and Confinement in NanoholeStructured Mesoscale Dielectric Spheres. Nanomaterials 2019, 9, 186

111. Minin, I.V., Minin, O.V., Cao, Y., Z. Liu, Y. Geints \& A. Karabchevsky. Optical vacuum cleaner by optomechanical manipulation of nanoparticles using nanostructured mesoscale dielectric cuboid. Sci Rep 2019, 9, 12748.

112. Minin, I.V. and Minin, O.V. Ultrafast all-optical THz modulation based on wavelength scaled dielectric particle with graphene monolayer. Proc. SPIE 11065, 2019, 110651J.

113. Minin, O. V.; Minin, I.V. and Cao, Y. Optical magnet for nanoparticles manipulations based on optical vacuum cleaner concept. Proc. SPIE 11845, 2021, 118451G.

114. Zhou, Y.; Hong, M. Formation of a three-dimensional bottle beam via an engineered microsphere. Photonics Research 2021, 9(8), 1598.

115. Dholakia, K. and Bruce, G. Optical hooks. Nature Photonics 2019, 13, 4229.

116. Minin, I.V, and Minin, O.V. Recent Trends in Optical Manipulation Inspired by Mesoscale Photonics and Diffraction Optics, J of Biomedical Photonics E Eng 2020, 6(2), 020301.

117. Minin, I.V, and Minin, O.V. Device for forming the optical trap in the form of the photonic hook, Patent of Russia 161207 (27 October, 2015).

118. Yue, L.; Minin, O.V.; Wang, Z.; Monks, J.; Snalin, A. and Minin, I.V. Photonic hook: A new curved light beam. Optics Letters 2018, 43(4), 771.

119. Spector, M.; Ang, A.; Minin, O. V.; Minin, I.V.; Karabchevsky, A. Temperature mediated 'Photonic hook' nanoparticle manipulator with pulsed illumination. Nanoscale Advances 2020, 2(6), 2595-2601.

120. Minin, I.V, and Minin, O.V. The Photonic Hook: From Optics to Acoustics and Plasmonics. Springer, Cham 2021.

121. Minin, I. V.; Minin, O.V.; Katyba, G.; Chernomyrdin, N.; Zaytsev, K.; Yue, L.; Wang, Z. and Christodoulides, D.N. Experimental observation of a photonic hook. APL, 2019, 114(3), 031105.

122. Spector, M.; Ang, A.; Minin, O. V.; Minin, I.V.; Karabchevsky, A. Photonic hook formation in near-infrared with MXene Ti3C2 nanoparticles. Nanoscale Advances 2020, 2, 5312-5318.

123. Gu, G; Shao, L; Song, J; Qu, J; Zheng, K; Shen, X. et al. Photonic hooks from Janus microcylinders. Opt Express 2019, 27, 37771-80.

124. Geints, Y.; Minin, I.V.; Minin, O.V. Tailoring 'photonic hook' from Janus dielectric microbar. J Opt 2020, $22,065606$.

125. Minin, I.V.; Minin, O.V. Dielectric particle-based strategy to design a new self-bending subwavelength structured light beams. IOP Conf. Series: Materials Science and Engineering, 2021, 1019, 012093.

126. Minin, I. V.; Minin, O.V.; Liu, C.; Wei, H.; Geints, Y.; Karabchevsky, A. Experimental demonstration of tunable photonic hook by partially illuminated dielectric microcylinder. Optics Letters 2020, 45(17), 4899.

127. Liu; C.; Chung, H.; Minin, O.V. and Minin, I.V. Shaping photonic hook via well-controlled illumination of finite-size graded-index micro-ellipsoid. Journal of optics 2020, 22(8), 085002.

128. Pacheco-Peña, V.; Riley, J.; Liu, C.; Minin, O.V. and Minin, I.V. Diffraction limited photonic hook via scattering and diffraction of dual-dielectric structures. Sci Rep 2021, 11, 20278. 
129. Ang, A.; Karabchevsky, A.; Minin, I. V.; Minin, O.V.; Sukhov, S. and Snalin, A. 'Photonic Hook' based optomechanical nanoparticle manipulator. Scientific Reports 2018, 8, 2029.

130. Ang, A.; Minin, I. V.; Minin, O.V.; Sukhov, S.;Snalin, A.; Karabchevsky, A. Low-contrast photonic hook manipulator for cellular differentiation. In META 2018, the 9th International Conference on Metamaterials, Photonic Crystals and Plasmonics. France, 2018

131. Cipolla MJ. The Cerebral Circulation. San Rafael (CA): Morgan \& Claypool Life Sciences; 2009. Chapter 6, Barriers of the CNS. Available from: www.ncbi.nlm.nih.gov/books/NBK53084/

132. Minin, I.V.; Minin, O.V.; Liu, Y.; Tuchin, V.; Liu, C. Concept of Photonic Hook Scalpel Generated by Shaped Fiber Tip with Asymmetric Radiation. J Biophotonics 2021, 14(2), e202000342.

133. Geints, Y.; Minin, O.V.; Yue, L. and Minin, I.V. Wavelength-scale photonic space switch proof-of-concept based on photonic hook effect. Ann. Phys. (Berlin) 2021, 533(9), 2100192.

134. Minin, I.V.; Shuvalov, G.V.; Minin, O.V. All-dielectric asymmetrical metasurfaces based on mesoscale dielectric particles with different optical transmissions in opposite directions through full internal reflection. In Frontier Research and Innovation in Optoelectronics Technology and Industry; CRC Press: Kunming, China, 2018; 437-440.

135. Liu, C.-Y.; Minin, O.V.; Minin, I.V. First experimental observation of array of photonic jets from saw-tooth phase diffraction grating. EPL (Europhys. Lett.) 2018, 123, 54003.

136. Ge, S.; Liu, W.; Zhang, J.; Huang, Y.; Xi, Y.; Yang, P.; Sun, X.; Li, S.; Lin, D.; Zhou, D.; Zhu, Y.; Li, W. and Yu, Y. Novel Bilayer Micropyramid Structure Photonic Nanojet for Enhancing a Focused Optical Field. Nanomaterials 2021, 11, 2034.

137. Balezin, M.; Baryshnikova, K.V.; Kapitanova, P.; Evlyukhin, A. Electromagnetic properties of the Great Pyramid: First multipole resonances and energy concentration. J. Appl. Phys. 2018, 124, 034903.

138. Minin, I.V.; Minin, O.V.; Yue, L. Electromagnetic properties of Pyramids from positions of photonics. Russ. Phys. J. 2020, $62,1763-1769$.

139. Schuller, J.; Barnard, E.; Cai, W.; Jun, Y.; White, J. and Brongersma, M. Plasmonics for extreme light concentration and manipulation. Nat. Mater. 2010, 9, 193.

140. Zhao, C.; Zhang, J. and Liu, Y. Light manipulation with encoded plasmonic nanostructures. EPJ Applied Metamaterials, 2014, 1,6 .

141. Ju, D.; Pei, H.; Jiang, Y.; and Sun Y, Controllable and enhanced nanojet effects excited by surface plasmon polariton. Appl. Phys. Lett. 2013, 102, 171109.

142. Pacheco-Pena, V.; Minin, I. V.; Minin, O.V.; Beruete, M. Comprehensive analysis of photonic nanojets in 3D dielectric cuboids excited by surface plasmons. Ann. Phys. 2016, 528, 684.

143. Pacheco-Pena, V.; Minin, I. V.; Minin, O.V.; Beruete, M. Increasing Surface Plasmons Propagation via Photonic Nanojets with Periodically Spaced 3D Dielectric Cuboids. Photonics 2016, 3, 10.

144. Minin, I. V.; Minin, O.V.; Glinskiy, I.; Malureanu, R.; Lavrinenko, A.; Yakubovsky, D.; Volcov, V.; Ponomarev, D. Plasmonic nanojet: an experimental demonstration. Optics Letters 2020, 45(12), 3244-3247.

145. Caldwell, J.; Lindsay, L.; Giannini, V.; Vurgaftman, I.; Reinecke, T.; Maier, S. and Glembocki, O. Low-loss, infrared and terahertz nanophotonics using surface phonon polaritons. Nanophotonics 2015, 4, 44-68.

146. Minin, I. V.; Minin, O.V.; Ponomarev, D.; Glinskiy, I. Photonic hook plasmons: A new curved surface wave. Ann. Phys. 2018, 530, 1800359.

147. Minin, I.V.; Minin, O.V.; Glinskiy, I.; Malureanu, R.; Lavrinenko, A.; Yakubovsky, D.; Volcov, V.; Ponomarev, D. Experimental verification of a plasmonic hook in a dielectric Janus particle. APL 2021, 118(13) 131107.

148. Zhang, Y.; Min, C.; Dou, X.; Wang, X.; Urbach, H.; Somekh, M. and Yuan, X. Plasmonic tweezers: for nanoscale optical trapping and beyond. Light: Science \& Applications 2021, 10, 59. 
149. Minin, O.V. and Minin, I.V. Acoustojet: acoustic analogue of photonic jet phenomenon based on penetrable 3D particle. Optical and Quantum Electronics 2017, 49-54.

150. Lopes, J.; Andrade, M.; Leao-Neto, J.; Adamowski, J.; Minin, I.V.; Silva, G. Focusing acoustic beams with a ball shaped lens beyond the diffraction limit, Phys. Rev. Appl. 2017, 8(2), 024013.

151. Minin, O.V. and Minin, I.V. Mesoscale acoustical cylindrical superlens. MATEC Web of Conferences, 2018, 155, 201815501029

152. Tarrazo-Serrano, D.; Rubio, C.; Minin, O.V.; Uris, A.; Minin, I.V. Ultrasonic focusing with mesoscale polymer cuboid. Ultrasonics 2020, 106, 106143.

153. Zhao, L.; Horiuchi, T. and Yu, M. Broadband ultra-long acoustic jet based on double-foci Luneburg lens. JASA Express Lett. 2021, 1, 114001.

154. Rubio, C.; Tarrazo-Serrano, D.; Minin, O.V.; Uris, A.; Minin, I.V. Enhancement of pupil masked wavelength-scale gasfilled flat acoustic lens based on anomaly apodization effect. Physics Letters A 2019, 383, 396-399.

155. Lu, C.; Yu, R.; Wang, K.; Wang, J.; and Wu, D. Tunable acoustic jet generated by a masked cylindrical lens. Applied Physics Express 2020, 13, 097003.

156. Minin, I. V.; Minin, O.V.; Tseplyaev, I. The relationship between resonance scattering and the formation of an acoustojet under the interaction of ultrasound with a dielectric sphere immersed in water. Journal of Physics: Conf. Series 2017, 881, 012025.

157. Rubio, C.; Minin, I. V.; Uris, A. and Minin, O.V. Super-resonances in a dielectric mesoscale sphere immersed in water: effects in extreme field localization of acoustic wave. Proc. Mtgs. Acoust. 2019, 38, 070001.

158. Rubio, C.; Tarrazo-Serrano, D.; Minin, O.V.; Uris, A.; Minin, I.V. Acoustical hooks: a new subwavelength self-bending beam. Results Phys 2020, 16, 102921.

159. Ren, X.; Zhou, Q.; Xu, Z.; Liu, X. Acoustic hook beam lens for particle trapping. Applied Physics Express 2020, $13,064003$.

160. Castiñeira-Ibáñez, S.; Tarrazó-Serrano, D.; Uris, A.; Rubio, C.; Minin, O.V. and Minin, I.V. Cylindrical 3D printed configurable ultrasonic lens for subwavelength focusing enhancement. Sci Rep 2020, 10, 20279.

161. Castiñeira-Ibáñez, S.; Tarrazó-Serrano, D.; Uris, A.; Rubio, C. Tunable acoustic hooks from Janus cylinder. Results in Physics 2021, 24, 104134.

162. Sarvazyan, A.; Urban, M. and Greenleaf, J. Acoustic waves in medical imaging and diagnostics, Ultrasound Med. Biol. 2013, 39(7), 1133-1146.

163. Gorgin, R.; Luo, Y. and Wu, Z. Environmental and operational conditions effects on Lamb wave based structural health monitoring systems: A review, Ultrasonics 2020, 105, 106114.

164. Wang, J.; Yurchenko, D.; Hu, G.; Zhao, L.; Tang, L. and Yang, Y. Perspectives in flow-induced vibration energy harvesting, Appl. Phys. Lett. 2021, 119(10), 100502.

165. Taylor M. A., Waleed M., Stilgoe A. B., Rubinsztein-Dunlop H. and Bowen W. P. Enhanced optical trapping via structured scattering, Nat. Photon. 2015, 9, 669-73

166. Dholakia, K. and Čižmár, T. Shaping the future of manipulation. Nat. Photonics 2011, 5, 335-42.

167. Hirano, K.; Shimizu, H.; Enami, T.; Terakawa, M.; Obara, M.; Nedyalkov, N. and Atanasov, P. Plasmonic Nanometric Optical Tweezers in an Asymmetric Space of Gold Nanostructured Substrates. J. of Nanotechnology in Diagnosis and Treatment, 2013, 1, 2-10.

168. Shi L., Meseguer F. Magnetic interaction in all silicon waveguide spherical coupler device. Opt. Express. 2012, 20, 2261622626.

169. Rastinehad, A.; Anastos, H.; Wajswol, E.; et al. Gold nanoshell-localized photothermal ablation of prostate tumors in a clinical pilot device study. PNAS, 2019, 116 (37), 18590-18596. 
170. Chen, L.; Zhou, Y.; Zhou, R. and Hong, M. Microsphere-Toward Future of Optical Microscopes. iScience 2020, $23,101211$.

171. Savo, R.; Morandi, A.; Muller, J.S.; Kaufmann, F.; Timpu, F.; Escale, M.R.; Zanini, M.; Isa, L. and Grange, R. Broadband Mie driven random quasi-phasematching. Nat. Photonics, 2020, 14, 740-747.

172. Huang, K.; Ye, H.; Teng, J.; Yeo, S.P.; Luk'yanchuk, B.; and Qiu, C.W. Optimization-free superoscillatory lens using phase and amplitude masks. Laser Photonics Rev., 2014, 8(1), 152-157.

173. Minin, I.V. and Minin, O.V. Control of focusing properties of diffraction elements. Sov. J. Quantum. Electron., 1990, 20(2), 198-199.

174. Mahariq, I.; Giden, I.; Minin, I.V.; Minin, O.V.; Kurt, H. Strong electromagnetic field localization near the surface of hemicylindrical particles. Opt. Quant. Electron., 2017, 49, 423.

175. Minin, I. V.; Minin, O.V.; Eremeev, A.; Tseplyaev, I. Spherical and cylindrical particle resonator as a cloak system. IOP Conf. Series: Materials Science and Engineering, 2018, 363, 012026.

176. Kravtsov, Yu.A. and Orlov, Yu.I. Limits of applicability of the method of geometric optics and related problems. Sov. Phys. Usp, 1980, 23, 750 . 